\title{
On global spatial regularity and convergence rates for time dependent elasto-plasticity
}

\author{
Dorothee Knees \\ Weierstrass Institute for Applied Analysis and Stochastics \\ Mohrenstr. 39, 10117 Berlin, Germany \\ E-Mail: knees@wias-berlin.de
}

\begin{abstract}
We study the global spatial regularity of solutions of generalized elasto-plastic models with linear hardening on smooth domains. Under natural smoothness assumptions on the data and the boundary we obtain $u \in L^{\infty}\left((0, T) ; H^{\frac{3}{2}-\delta}(\Omega)\right)$ for the displacements and $z \in L^{\infty}\left((0, T) ; H^{\frac{1}{2}-\delta}(\Omega)\right)$ for the internal variables. The proof relies on a reflection argument which gives the regularity result in directions normal to the boundary on the basis of tangential regularity results. Based on the regularity results we derive convergence rates for a finite element approximation of the models.
\end{abstract}

Mathematics Subject Classification (2000) 35B65, 49N60, 74C05, 74C10, 74S05

Keywords: elasto-plasticity, visco-plasticity, global regularity, reflection argument, convergence rate

\section{Introduction}

This paper is devoted to the study of global spatial regularity properties of solutions to elasto-plastic models in a geometrically linear framework. The regularity results then are applied to derive the convergence rate for a Finite Element discretization of the elastoplastic models. The model class under consideration comprises rate independent elastoplasticity with kinematic hardening combined with a von Mises flow rule or a Tresca flow rule, as well as elasto-visco-plastic models which include Cosserat effects.

Let $\Omega \subset \mathbb{R}^{d}$ be a bounded domain which represents an elasto-(visco)-plastic body and let $S=(0, T)$ be a time interval. The behavior of the body under the influence of external 
loadings is characterized by the (generalized) displacements $u: S \times \Omega \rightarrow \mathbb{R}^{m}$ and a vector of internal variables $z: S \times \Omega \rightarrow \mathbb{R}^{n}$, which represent the plastic strains and further hardening variables. The time evolution under the influence of external forces is determined through the quasi-static balance of forces (1.1) and an evolution law for the internal variable (1.2). The resulting model consists of a system of linear elliptic partial differential equations for $u$ which is strongly coupled with an evolution inclusion for $z$ :

$$
\begin{aligned}
\operatorname{div}(C(x) \nabla u(t, x)+B(x) z(t, x))+f(t, x)=0 & \text { for }(t, x) \in S \times \Omega, \\
\partial_{t} z(t, x) \in g\left(-\left(B^{\top}(x) \nabla u(t, x)+L(x) z(t, x)\right)\right) & \text { for }(t, x) \in S \times \Omega, \\
z(0, x)=z_{0}(x) & \text { for } x \in \Omega
\end{aligned}
$$

together with boundary conditions for $u$. The underlying stored elastic energy is given by

$$
\mathcal{E}(u, z)=\int_{\Omega} \frac{1}{2}\left\langle\left(\begin{array}{cc}
C & B \\
B^{\top} & L
\end{array}\right)\left(\begin{array}{c}
\nabla u \\
z
\end{array}\right),\left(\begin{array}{c}
\nabla u \\
z
\end{array}\right)\right\rangle \mathrm{d} x
$$

with a symmetric coefficient tensor $A=\left(\begin{array}{cc}C & B \\ B^{\top} & L\end{array}\right) \in L^{\infty}\left(\Omega ; \operatorname{Lin}\left(\mathbb{R}^{m \times d} \times \mathbb{R}^{n}, \mathbb{R}^{m \times d} \times \mathbb{R}^{n}\right)\right)$. Moreover, $g: \mathbb{R}^{n} \rightarrow \mathcal{P}\left(\mathbb{R}^{n}\right)$ is a monotone multivalued constitutive function. If $\mathcal{E}$ is positive semi-definite and if $0 \in g(0)$, then the system (1.1)-(1.3) belongs to the class of models of monotone type introduced in [Alb98], which is a generalization of the class of generalized standard materials. With the choice $g=\partial \chi_{K}$, where $\partial \chi_{K}$ is the subdifferential of the characteristic function $\chi_{K}$ related to the convex set $K \subset \mathbb{R}^{n}$, equations (1.1)-(1.3) describe classical rate-independent elasto-plasticity. The set $K$ is the set of admissible generalized stresses. A more detailed definition of the model is given in Section 4 .

If the elastic energy $\mathcal{E}$ is coercive, i.e. if

$$
\mathcal{E}(u, z) \geq \frac{\alpha}{2}\left(\|u\|_{H^{1}(\Omega)}^{2}+\|z\|_{L^{2}(\Omega)}^{2}\right)
$$

for all $u \in H_{0}^{1}(\Omega)$ and $z \in L^{2}(\Omega)$ and some constant $\alpha>0$, then classical results guarantee the existence of a unique pair $(u, z) \in W^{1,1}\left(S ; H^{1}(\Omega)\right) \times W^{1,1}\left(S ; L^{2}(\Omega)\right)$ which solves (1.1)(1.3), see e.g. [DL72, Joh78, Bré73, HHLN88, HR99, AC04] and the references therein.

The main result of our paper is Theorem 4.1, where we prove the following global spatial regularity for $(u, z)$ provided that $\partial \Omega$ is smooth, that $\mathcal{E}$ is coercive, that the type of the boundary conditions does not change and that the data and coefficients have some natural smoothness properties: For all $\delta>0$ it holds

$$
\begin{aligned}
& u \in L^{\infty}\left(S ; H^{\frac{3}{2}-\delta}(\Omega)\right), \\
& z \in L^{\infty}\left(S ; H^{\frac{1}{2}-\delta}(\Omega)\right),
\end{aligned}
$$


where $H^{s}(\Omega)$ stands for Sobolev-Slobodeckij spaces, see e.g. [Tri83]. This result was announced in the paper [Kne09], where we studied a model problem on a cube.

The intrinsic difficulty of proving spatial regularity for time-dependent plasticity problems stems from the fact that the flow rule (1.2) is nonsmooth and has no regularizing terms. Hence, spatial regularity has to be maintained during the evolution by careful estimates. Let $\mathcal{Q} \subset H^{1}(\Omega) \times L^{2}(\Omega) \ni(u(t), z(t))$ denote the state space. The main problem is that the data to solution map is not Lipschitz as a mapping from $W^{1,1}\left(S ; \mathcal{Q}^{*}\right) \rightarrow W^{1,1}(S ; \mathcal{Q})$, but only as a mapping from $W^{1,1}\left(S ; \mathcal{Q}^{*}\right) \rightarrow L^{\infty}(S ; \mathcal{Q})$, see Theorem 2.2 . This stability estimate is the basis for proving local and tangential results of the type $\nabla u, z \in L^{\infty}\left(S ; H_{\text {loc }}^{1}(\Omega)\right)$. Since a similar Lipschitz estimate is not available for the time derivative, one cannot derive a spatial regularity result of the type $\partial_{t} z \in L^{\infty}\left(S ; H_{\mathrm{loc}}^{1}(\Omega)\right)$. Indeed, the example in Section 5.2.4 shows that the latter regularity in general is not valid in spite of smooth data. Since terms of the form $\partial_{\operatorname{tang}} z \in L^{\infty}\left(S ; L^{2}(\Omega)\right)$ enter as data when we prove the regularity in normal direction, we cannot apply the aforementioned Lipschitz estimate any more since it would require $\partial_{\operatorname{tang}} z \in W^{1,1}\left(S ; L^{2}(\Omega)\right)$. In this situation we only have a weaker Hölder estimate with exponent $\frac{1}{2}$ for the solution to data map, see Theorem 2.2. This explains, why in the normal direction we obtain a "half" spatial derivative, only.

The essential argument in this paper is to apply a reflection argument in order to obtain higher differentiability properties for $\nabla u$ and $z$ for the direction perpendicular to the boundary, see Section 3.3. After localizing system (1.1)-(1.3) to a half cube by the usual techniques, we reflect the problem to the full cube using an even extension for the internal variable $z$ and an odd extension for the displacements modified by the value of $u$ on the boundary. We show that the newly defined functions satisfy a problem of the type (1.1)-(1.3) on the full cube with coefficients depending smoothly on the space variable. The right hand side of the extended problem contains tangential derivatives of $\nabla u$ and $z$. Using tangential regularity results and the Hölder property of the data to solution map, we obtain the additional "half" spatial derivative.

It is an unsolved problem, whether our final result (1.4)-(1.5) is optimal or whether one should expect $u \in L^{\infty}\left(S ; H^{2}(\Omega)\right)$. This would coincide nicely with the local and tangential properties $\nabla u, z \in L^{\infty}\left(S ; H_{\mathrm{loc}}^{1}(\Omega)\right)$ and also with results for solutions of linear elliptic equations on smooth domains. Our global result (1.4)-(1.5) can be improved in a special scalar case, i.e. $m=1$. Under strong coupling assumptions between the coefficient matrices $C, B, L$ and the function $g$, we obtain indeed the full spatial regularity $u \in L^{\infty}\left(S ; H^{2}(\Omega)\right)$, see Section 5.2.3. Here, we use a reflection argument, which takes into account the explicit structure of the coefficient matrix $A=\left(\begin{array}{cc}C & B \\ B^{\top} & L\end{array}\right)$. This result is also extended to cubes.

In the final section we derive an estimate for the error between the solution of (1.1)-(1.3) 
and discrete solutions which are obtained from a finite element discretization in space and an implicit Euler scheme in time. Using the above proved regularity results we obtain an estimate for the convergence rate without any additional assumption on the smoothness of the solutions.

Let us give a short discussion of regularity results in the literature for systems of the type (1.1)-(1.3). Recently, the question of global spatial regularity attracted much attention. We mention here the contributions by Alber/Nesenenko [AN09] and by Frehse/Löbach [FL08b]. In [AN09] the authors obtain for a model similar to (1.4)-(1.5) the global result $u \in L^{\infty}\left(S ; H^{1+\frac{1}{3}-\delta}(\Omega)\right)$ and $z \in L^{\infty}\left(S ; H^{\frac{1}{3}-\delta}(\Omega)\right)$ by first proving a local result and the tangential result $\partial_{\operatorname{tang}} \nabla u \in L^{\infty}\left(S ; L^{2}(\Omega)\right)$. They show that this already implies that $u \in$ $L^{\infty}\left(S ; H^{1+\frac{1}{4}-\delta}(\Omega)\right)$, and similarly for $z$. By an iteration procedure they improve then the differentiability from $\frac{1}{4}$ to $\frac{1}{3}$. In the paper [FL08b] the authors study regularity properties of rate independent elasto-plastic models with a von Mises flow rule and linear kinematic or isotropic hardening. They show Hölder regularity of the stresses up to the boundary, derive the spatial regularity $\nabla \sigma \in L^{\infty}\left(S ; L^{1+\delta}(\Omega)\right)$ for the stress $\sigma$ and prove several additional integrability properties. The investigations take a stress based version of (1.4)-(1.5) as a starting point.

Local regularity properties for the model in (1.4)-(1.5) and variants of it, having e.g. only a positive semi-definite elastic energy, were investigated by several authors [BF96, FL08a, Shi99, Ser92, Dem09, Dem08, NC08]. Here, one typically finds that the stress $\sigma=C \nabla u+$ $B z$ belongs to $L^{\infty}\left(S ; H_{\text {loc }}^{1}(\Omega)\right)$. Similar results are valid for $u$ and $z$ provided that the elastic energy $\mathcal{E}$ is coercive.

Further global results are available for time discretized versions of (1.4)-(1.5), see for example [Rep96, KN08] and the references therein. Here one obtains $\sigma\left(t_{k}\right) \in H^{1}(\Omega)$ globally for smooth domains and smooth data at every temporal discretization point $t_{k}$. However, up to now it is to our knowledge an open question whether a uniform estimate of the form $\sup _{\text {time step } \Delta t>0, k \Delta t \leq T}\|\sigma(k \Delta t)\|_{H^{1}(\Omega)} \leq c$ is valid. This estimate would allow to carry over the result from the discretized model to the continuous one. Finally, for the stationary Hencky model of perfect plasticity we have the global result $\sigma \in H^{\frac{1}{2}-\delta}(\Omega), \delta>0$, for domains with Lipschitz boundary and with changing boundary conditions, [Kne06].

\section{Existence results and stability estimates}

In this section abstract existence results and stability estimates for problems of the type (1.1)-(1.3) are recalled. The results are based on classical existence theorems by Brézis 
[Bré73] for evolution equations with maximal monotone operators. We also refer to [AC04, HR99] and the references therein for the discussion of particular elastic-plastic and viscoplastic models. Furthermore, we provide an estimate for the error between the solution and approximations calculated from a time-discretized version on subspaces of $\mathcal{Z}$. The estimate is valid under the same assumptions as in the existence theorem and no additional regularity properties of the solutions are needed. Using the regularity results, which we derive in Sections 3-5, convergence rates will be deduced in Section 6 .

Let $S=(0, T)$ be the time interval and $\mathcal{Q}$ a Hilbert space. By $W^{k, p}(S ; \mathcal{Q}), p \in[1, \infty]$, $k \in \mathbb{N}$, we denote the Sobolev space of Bochner integrable functions having $p$-integrable weak derivatives up to order $k$.

\subsection{Existence result and stability estimates}

By $\mathcal{Q}=\mathcal{U} \times \mathcal{Z}$ we denote the state spaces which is composed of the real, separable Hilbert spaces $\mathcal{U}$ and $\mathcal{Z}$. We identify the dual $\mathcal{Z}^{*}$ with $\mathcal{Z}$ but distinguish between $\mathcal{U}$ and the dual space $\mathcal{U}^{*}$. For $u \in \mathcal{U}$ and $z \in \mathcal{Z}$ the stored energy is given by the following quadratic functional

$$
\mathcal{E}(u, z)=\frac{1}{2}\langle\mathcal{A}(u, z),(u, z)\rangle,
$$

where $\langle\cdot, \cdot\rangle$ stands for the dual pairing in $\mathcal{Q}^{*} \times \mathcal{Q}$. It is assumed that $\mathcal{A} \in \operatorname{Lin}\left(\mathcal{Q}, \mathcal{Q}^{*}\right)$ is a linear, bounded and self adjoint operator and that there exists a constant $\alpha>0$ such that

$$
\mathcal{E}(u, z) \geq \frac{\alpha}{2}\left(\|u\|_{\mathcal{U}}^{2}+\|z\|_{\mathcal{Z}}^{2}\right)
$$

for all $(u, z) \in \mathcal{Q}$. Let furthermore $\mathcal{G}: \mathcal{Z} \rightarrow \mathcal{P}(\mathcal{Z})$ be a maximal monotone operator with $0 \in \mathcal{G}(0)$.

For given data $z_{0} \in \mathcal{Z}$ and $\ell=\left(\ell_{1}, \ell_{2}\right): S \rightarrow \mathcal{Q}^{*}$ the problem under consideration is: Find $u: S \rightarrow \mathcal{U}, z: S \rightarrow \mathcal{Z}$ with $z(0)=z_{0}$ such that for a.e. $t \in S$

$$
\begin{aligned}
D_{u} \mathcal{E}(u(t), z(t)) & =\ell_{1}(t) \\
\partial_{t} z(t) & \in \mathcal{G}\left(-D_{z} \mathcal{E}(u(t), z(t))+\ell_{2}(t)\right) .
\end{aligned}
$$

We call the data $z_{0}$ and $\ell$ compatible if there exists $u_{0} \in \mathcal{U}$ with $D_{u} \mathcal{E}\left(u_{0}, z_{0}\right)=\ell_{1}(0)$ and with $-D_{z} \mathcal{E}\left(u_{0}, z_{0}\right)+\ell_{2}(0) \in D(\mathcal{G})$, where $D(\mathcal{G})$ denotes the domain of $\mathcal{G}$.

The following existence theorem is classical for evolution inclusions with maximal monotone operators. 
Theorem 2.1. Under the above assumptions there exists for every compatible data $\ell \in$ $W^{2,1}\left(S ; \mathcal{Q}^{*}\right), z_{0} \in \mathcal{Z}$ a unique pair $(u, z) \in W^{1, \infty}(S ; \mathcal{Q})$ which solves $(2.2)$.

If $\mathcal{G}=\partial \chi_{\mathcal{K}}$, where $\mathcal{K} \subset \mathcal{Z}$ is convex, closed and with $0 \in \mathcal{K}$, then for every compatible data $\left(z_{0}, \ell\right) \in \mathcal{Z} \times W^{1,1}\left(S ; \mathcal{Q}^{*}\right)$ there exists a unique pair $(u, z) \in W^{1,1}(S ; \mathcal{Q})$ solving $(2.2)$.

In order to fix the notation, we give here a short sketch of the proof of Theorem 2.1.

Proof. The linear operator $\mathcal{A}$ is split as follows

$$
\mathcal{A}(u, z)=\left(\begin{array}{ll}
\mathcal{A}_{11} & \mathcal{A}_{12} \\
\mathcal{A}_{21} & \mathcal{A}_{22}
\end{array}\right)\left(\begin{array}{l}
u \\
z
\end{array}\right)
$$

with bounded operators $\mathcal{A}_{11} \in \operatorname{Lin}\left(\mathcal{U}, \mathcal{U}^{*}\right), \mathcal{A}_{12} \in \operatorname{Lin}\left(\mathcal{Z}, \mathcal{U}^{*}\right), \mathcal{A}_{21}=\mathcal{A}_{12}^{*} \in \operatorname{Lin}(\mathcal{U}, \mathcal{Z})$ and $\mathcal{A}_{22} \in \operatorname{Lin}(\mathcal{Z}, \mathcal{Z})$. By $\mathcal{L}: \mathcal{Z} \rightarrow \mathcal{Z}$ we denote the Schur complement operator associated with $\mathcal{A}$, i.e. $\mathcal{L}=\mathcal{A}_{22}-\mathcal{A}_{21} \mathcal{A}_{11}^{-1} \mathcal{A}_{12}$. The assumptions on $\mathcal{A}$ imply that $\mathcal{L}$ is a bounded, self adjoint operator with $\langle\mathcal{L} z, z\rangle \geq \alpha\|z\|_{\mathcal{Z}}^{2}$ for all $z \in \mathcal{Z}$ and $\alpha$ from (2.1). Problem (2.2) is equivalent to the following reduced version: Find $z: S \rightarrow \mathcal{Z}$ with

$$
\partial_{t} z(t) \in \mathcal{G}(-\mathcal{L} z(t)+F(t)), \quad z(0)=z_{0},
$$

where $F(t)=\ell_{2}(t)-\mathcal{A}_{21} \mathcal{A}_{11}^{-1} \ell_{1}(t)$. Now $u$ can be calculated via $u=\mathcal{A}_{11}^{-1}\left(\ell_{1}-\mathcal{A}_{12} z\right)$.

In terms of the new variable $y(t)=\mathcal{L}^{\frac{1}{2}} z(t)-\mathcal{L}^{-\frac{1}{2}} F(t)$, the mapping $\widetilde{\mathcal{G}}: \mathcal{Z} \rightarrow \mathcal{P}(\mathcal{Z})$ with $\widetilde{\mathcal{G}}(\zeta)=\mathcal{L}^{\frac{1}{2}} \mathcal{G}\left(\mathcal{L}^{\frac{1}{2}} \zeta\right)$ and the data $f(t)=-\mathcal{L}^{-\frac{1}{2}} \partial_{t} F(t)$, problem $(2.3)$ can equivalently be written as: Find $y: S \rightarrow \mathcal{Z}$ with

$$
\partial_{t} y(t) \in f(t)+\widetilde{\mathcal{G}}(-y(t)), \quad y(0)=y_{0}=\mathcal{L}^{\frac{1}{2}} z_{0}-\mathcal{L}^{-\frac{1}{2}} F(0) .
$$

The operator $\widetilde{\mathcal{G}}$ is maximal monotone with respect to the standard scalar product in $\mathcal{Z}$.

Theorem 3.4 and Proposition 3.3 in [Bré73] applied to (2.4) provide the existence result in case of an arbitrary maximal monotone mapping $\mathcal{G}$, while Proposition 3.4 from [Bré73] gives the result for the case $\mathcal{G}=\partial \chi_{\mathcal{K}}$.

In the next Theorem we recall stability estimates which are the basis for our regularity results. For more detailed continuity properties of the solution operator we refer to [Kre99].

Theorem 2.2. Assume (2.1) and let $\mathcal{G}: \mathcal{Z} \rightarrow \mathcal{P}(\mathcal{Z})$ be a monotone operator.

(a) There exists a constant $\kappa_{1}>0$ such that for all $u^{i} \in L^{\infty}(S ; \mathcal{U})$ and $z^{i} \in W^{1,1}(S ; \mathcal{Z})$, $i \in\{1,2\}$, which are solutions to problem (2.2) with data $z_{0}^{i} \in \mathcal{Z}$ and $\ell^{i}=\left(\ell_{1}^{i}, \ell_{2}^{i}\right) \in$ 


$$
\begin{aligned}
& L^{\infty}\left(S ; \mathcal{Q}^{*}\right), \text { it holds } \\
& \quad\left\|u^{1}-u^{2}\right\|_{L^{\infty}(S ; \mathcal{U})}^{2}+\left\|z^{1}-z^{2}\right\|_{L^{\infty}(S ; \mathcal{Z})}^{2} \\
& \quad \leq \kappa_{1}\left(\left\|z_{0}^{1}-z_{0}^{2}\right\|_{\mathcal{Z}}^{2}+\left\|z^{1}-z^{2}\right\|_{W^{1,1}(S ; \mathcal{Z})}\left\|\ell^{1}-\ell^{2}\right\|_{L^{\infty}\left(S ; \mathcal{Q}^{*}\right)}+\left\|\ell_{1}^{1}-\ell_{1}^{2}\right\|_{L^{\infty}\left(S ; \mathcal{U}^{*}\right)}^{2}\right)
\end{aligned}
$$

(b) There exists a constant $\kappa_{2}>0$ such that for all $u^{i} \in W^{1,1}(S ; \mathcal{U})$ and $z^{i} \in W^{1,1}(S ; \mathcal{Z})$, which are solutions to problem (2.2) with respect to the data $z_{0}^{i} \in \mathcal{Z}$ and $\ell^{i} \in$ $W^{1,1}\left(S ; \mathcal{Q}^{*}\right)$, it holds

$$
\left\|u^{1}-u^{2}\right\|_{L^{\infty}(S ; \mathcal{U})}+\left\|z^{1}-z^{2}\right\|_{L^{\infty}(S ; \mathcal{Z})} \leq \kappa_{2}\left(\left\|z_{0}^{1}-z_{0}^{2}\right\|_{\mathcal{Z}}+\left\|\ell^{1}-\ell^{2}\right\|_{W^{1,1}\left(S ; \mathcal{Q}^{*}\right)}\right) .
$$

Proof. Assumption (2.1) implies that there exists $\kappa>0$ such that

$$
\left\|u^{1}-u^{2}\right\|_{L^{\infty}(S ; \mathcal{U})} \leq \kappa\left(\left\|z^{1}-z^{2}\right\|_{L^{\infty}(S ; \mathcal{Z})}+\left\|\ell_{1}^{1}-\ell_{1}^{2}\right\|_{L^{\infty}\left(S ; \mathcal{U}^{*}\right)}\right)
$$

Let $\mathcal{L}$ be the Schur complement operator and $F^{i}, i \in\{1,2\}$, be the functions defined in the proof of Theorem 2.1. Since $\mathcal{G}$ is monotone and since $\mathcal{L}$ is self adjoint, the solutions $z^{i}$ of (2.3) satisfy for almost every $t \in S$

$$
\frac{1}{2} \frac{\mathrm{d}}{\mathrm{d} t}\left\langle z^{1}(t)-z^{2}(t), \mathcal{L}\left(z^{1}(t)-z^{2}(t)\right)\right\rangle \leq\left\langle\partial_{t}\left(z^{1}(t)-z^{2}(t)\right), F^{1}(t)-F^{2}(t)\right\rangle .
$$

Integrating this estimate with respect to $t$, applying Hölder's inequality and taking into account (2.7) results in (2.5).

If $\ell \in W^{1,1}\left(S ; \mathcal{Q}^{*}\right)$, then integrating (2.8) with respect to $t$, partial integration and Young's inequality result in the estimate

$$
\begin{aligned}
& \left\|z^{1}(t)-z^{2}(t)\right\|_{\mathcal{Z}}^{2} \leq c\left(\left\|z_{0}^{1}-z_{0}^{2}\right\|_{\mathcal{Z}}^{2}+\int_{0}^{t}\left\|z^{1}(s)-z^{2}(s)\right\|_{\mathcal{Z}}^{2} \mathrm{~d} s\right. \\
& \left.\quad+\left\|F^{1}-F^{2}\right\|_{W^{1,1}(S ; \mathcal{Z})}\left(\left\|F^{1}-F^{2}\right\|_{W^{1,1}(S ; \mathcal{Z})}+\left\|z^{1}-z^{2}\right\|_{L^{\infty}(S ; \mathcal{Z})}\right)\right) .
\end{aligned}
$$

Applying the Gronwall inequality and Young's inequality to the previous estimate leads in combination with (2.7) to estimate (2.6).

\subsection{An estimate of the approximation error}

We adopt the notation of the previous Section 2.1. The goal of this section is to derive an abstract error estimate for the approximation of the solution of (2.3) by time-discretized 
solutions on subspaces of $\mathcal{Z}$. Here, we restrict ourselves to the case where $\mathcal{G}$ is the subdifferential of the characteristic function of a convex, closed set $\mathcal{K} \subset \mathcal{Z}$. In this case the corresponding evolution model describes a rate independent process and we may directly apply Theorem 2.2 from [MPPS09] in order to estimate the error due to time-discretization.

For $h \in\left[0, h_{0}\right]$ let $\mathcal{Z}_{h} \subset \mathcal{Z}$ be closed subspaces, $\mathcal{Z}_{0}=\mathcal{Z}$, and let $\mathcal{L}_{h}: \mathcal{Z} \rightarrow \mathcal{Z}$ be linear, uniformly bounded and self adjoint operators which are uniformly positive definite on $\mathcal{Z}$ (not only on $\mathcal{Z}_{h}$ ), i.e.

$$
\sup _{0 \leq h \leq h_{0}}\left\|\mathcal{L}_{h}\right\|_{\text {op }} \leq \beta \text { and for all } z \in \mathcal{Z} \text { we have }\left\langle\mathcal{L}_{h} z, z\right\rangle \geq \alpha\|z\|^{2}
$$

for some $\alpha, \beta>0$, which are independent of $h$. In addition it is assumed that $\mathcal{L}_{h}: \mathcal{Z}_{h} \rightarrow \mathcal{Z}_{h}$ for all $h$. Later, for $h>0$ the operators $\mathcal{L}_{h}$ will be identified with discretized versions of the Schur complement operator $\mathcal{L} \equiv \mathcal{L}_{0}$ introduced in the previous section.

Let $\mathcal{G}: \mathcal{Z} \rightarrow \mathcal{P}(\mathcal{Z})$ be given as $\mathcal{G}=\partial_{\mathcal{Z}} \chi_{\mathcal{K}}$, where $\mathcal{K} \subset \mathcal{Z}$ is closed, convex and $0 \in \mathcal{K}$ and $\partial_{\mathcal{Z}}$ denotes the subdifferential of $\chi_{\mathcal{K}}$ with respect to the space $\mathcal{Z}$. We make the following compatibility assumption on the spaces $\mathcal{Z}_{h}$ and the operator $\mathcal{G}$ :

$$
\text { for all } \eta \in \mathcal{Z}_{h} \text { it holds } \mathcal{G}(\eta) \cap \mathcal{Z}_{h}=\partial_{\mathcal{Z}_{h}} \chi_{\mathcal{K}_{h}}(\eta)
$$

where $\mathcal{K}_{h}=\mathcal{K} \cap \mathcal{Z}_{h}$ and $\partial_{\mathcal{Z}_{h}}$ is the subdifferential with respect to $\mathcal{Z}_{h}$. We define $\mathcal{G}_{h}: \mathcal{Z}_{h} \rightarrow$ $\mathcal{P}\left(\mathcal{Z}_{h}\right)$ as $\mathcal{G}_{h}=\partial_{\mathcal{Z}_{h}} \chi_{\mathcal{Z}_{h}}$. Obviously, $\mathcal{G}_{0}=\mathcal{G}$ and $\mathcal{G}_{h}(\eta) \subset \mathcal{G}(\eta)$ for $\eta \in \mathcal{Z}_{h}$.

For given $F_{h} \in W^{1,1}\left(S ; \mathcal{Z}_{h}\right)$ and $z_{0, h} \in \mathcal{Z}_{h}$ consider the problem: Find $z_{h} \in W^{1,1}\left(S ; \mathcal{Z}_{h}\right)$ with $z_{h}(0)=z_{0, h}$ such that for a.e. $t \in S$

$$
\partial_{t} z_{h}(t) \in \mathcal{G}_{h}\left(-\mathcal{L}_{h} z_{h}(t)+F_{h}(t)\right)
$$

Like in the previous section we call $\left(z_{0, h}, F_{h}\right)$ compatible if $-\mathcal{L}_{h} z_{0, h}+F_{h}(0) \in D\left(\mathcal{G}_{h}\right)$.

Theorem 2.3. Assume that for all $h \in\left[0, h_{0}\right]$ the operators $\mathcal{L}_{h}$ and $\mathcal{G}_{h}$ have the above described properties. Let further the functions $z_{h} \in W^{1,1}\left(S ; \mathcal{Z}_{h}\right)$ satisfy $(2.12)$ with compatible data $F_{h} \in W^{1,1}\left(S ; \mathcal{Z}_{h}\right)$ and $z_{0, h} \in \mathcal{Z}_{h}$. Then for all $t \in S$ it holds

$$
\begin{aligned}
& \alpha\left\|z(t)-z_{h}(t)\right\|_{\mathcal{Z}}^{2} \\
& \leq \beta\left\|z_{0,0}-z_{0, h}\right\|_{\mathcal{Z}}^{2}+2\left\|\partial_{t} z-\partial_{t} z_{h}\right\|_{L^{1}(S ; \mathcal{Z})}\left\|\left(\mathcal{L}_{h} z-F_{h}\right)-\left(\mathcal{L}_{0} z-F_{0}\right)\right\|_{L^{\infty}(S ; \mathcal{Z})},
\end{aligned}
$$

where $z(t):=z_{h=0}(t)$.

By Theorem 2.1 the existence of the functions $z$ and $z_{h}$ is guaranteed. 
Proof. Observe that $z$ satisfies (2.12) for $h=0$ if and only if for almost all $t$ it holds

$$
\left\langle\partial_{t} z(t)-\theta,\left(-\mathcal{L}_{0} z(t)+F_{0}(t)\right)-\eta\right\rangle \geq 0 \quad \forall \eta \in \mathcal{Z}, \theta \in \mathcal{G}(\eta) .
$$

In view of $(2.11)$ this inequality is valid for $\theta=\partial_{t} z_{h}(t) \in \mathcal{G}_{h}\left(-\mathcal{L}_{h} z_{h}(t)+F_{h}(t)\right)$. Hence, using the symmetry of $\mathcal{L}_{h}$ it follows for a.e. $t \in S$ :

$$
\begin{aligned}
\frac{\mathrm{d}}{\mathrm{d} t} \frac{1}{2}\left\langle\mathcal{L}_{h}\left(z-z_{h}\right)(t),\left(z-z_{h}\right)(t)\right\rangle \\
=\left\langle\partial_{t}\left(z-z_{h}\right)(t), \mathcal{L}_{0} z(t)-F_{0}(t)+\left(F_{h}(t)-\mathcal{L}_{h} z_{h}(t)\right)\right\rangle \\
\quad+\left\langle\partial_{t}\left(z-z_{h}\right)(t), F_{0}(t)-\mathcal{L}_{0} z(t)-\left(F_{h}(t)-\mathcal{L}_{h} z(t)\right)\right\rangle
\end{aligned}
$$

Due to (2.14) the first term on the right hand side is not positive. After integration with respect to $t$ and using the uniform positivity and boundedness of $\mathcal{L}_{h}$ we arrive at (2.13).

Next we investigate the error between the solution of (2.12) and the solutions of the fully discretized problems.

For the time discretization an implicit Euler scheme is used. Let $\tau \in[0, T]$ denote the fineness of the partition $\Pi^{\tau}=\left\{0=t_{\tau}^{0}<t_{\tau}^{1}<\ldots<t_{\tau}^{N_{\tau}}=T\right\}$ of the time interval $[0, T]$, i.e. $\tau=\max \left\{t_{\tau}^{i}-t_{\tau}^{i-1} ; 1 \leq i \leq N_{\tau}\right\}$. The fully discretized problem reads: For $1 \leq k \leq N_{\tau}$ find $z_{h, \tau}^{k} \in \mathcal{Z}_{h}$ such that with $z_{h, \tau}^{0}=z_{0, h}$ we have

$$
\frac{z_{h, \tau}^{k}-z_{h, \tau}^{k-1}}{t_{\tau}^{k}-t_{\tau}^{k-1}} \in \mathcal{G}_{h}\left(-\mathcal{L}_{h}\left(z_{h, \tau}^{k}\right)+F_{h}\left(t_{\tau}^{k}\right)\right) .
$$

By $\hat{z}_{h, \tau}: S \rightarrow \mathcal{Z}_{h}$ we denote the continuous and piecewise affine interpolant corresponding to $\left\{z_{h, \tau}^{k} ; 0 \leq k \leq N_{\tau}\right\}$.

Let $\mathcal{R}: \mathcal{Z} \rightarrow \mathbb{R}_{\infty}=\mathbb{R} \cup\{\infty\}$ be the convex conjugate functional to $\chi_{K}$, i.e. $\mathcal{R}(\theta)=$ $\sup _{\eta \in \mathcal{K}}\langle\eta, \theta\rangle$, and let $\mathcal{R}_{h}: \mathcal{Z}_{h} \rightarrow \mathbb{R}_{\infty}$ be the convex conjugate to $\chi_{\mathcal{K}_{h}}$ with respect to $\mathcal{Z}_{h}$. Observe that $\mathcal{R}$ and $\mathcal{R}_{h}$ are convex, lower semicontinuous and positively homogeneous of degree one, [IT79]. Moreover, the compatibility assumption (2.11) implies that $\mathcal{R}_{h}=\left.\mathcal{R}\right|_{\mathcal{Z}_{h}}$. Hence, problem (2.15) is equivalent to the following minimization problem

$$
z_{h, \tau}^{k} \in \operatorname{Argmin}\left\{\frac{1}{2}\left\langle\mathcal{L}_{h} \eta, \eta\right\rangle-\left\langle F_{h}\left(t_{\tau}^{k}\right), \eta\right\rangle+\mathcal{R}\left(\eta-z_{h, \tau}^{k-1}\right) ; \eta \in \mathcal{Z}_{h}\right\} .
$$

The existence of minimizers and the solvability of the discrete problem (2.15) follows with direct method in the calculus of variations. Due to the positivity assumptions on $\mathcal{L}_{h}$ the minimizer is unique.

The error estimate between the solution of the spatially discretized problem (2.12) and the fully discretized problem relies on a straightforward adaption of Theorem 2.2 in [MPPS09]. The important point is that the estimate holds uniformly with respect to the spaces $\mathcal{Z}_{h}, h \in\left[0, h_{0}\right]$. 
Theorem 2.4. [MPPS09] Let the assumptions of Theorem 2.3 be satisfied and assume that for all $h \in\left[0, h_{0}\right]$ the data $\left(z_{0, h}, F_{h}\right) \in \mathcal{Z}_{h} \times W^{1, \infty}\left(S ; \mathcal{Z}_{h}\right)$ are compatible with $\sup _{0 \leq h \leq h_{0}}\left(\left\|z_{0, h}\right\|_{\mathcal{Z}}+\left\|F_{h}\right\|_{W^{1, \infty}(S ; \mathcal{Z})}\right)<\infty$. Let $z_{h} \in W^{1,1}\left(S ; \mathcal{Z}_{h}\right)$ be the solution of $(2.12)$ and let $\hat{z}_{h, \tau}$ be the above introduced affine interpolant. Then there exists a constant $c>0$ such that for all $t, \tau \in S$ and all $h \in\left[0, h_{0}\right]$ it holds

$$
\left\|z_{h}(t)-\hat{z}_{h, \tau}(t)\right\|_{\mathcal{Z}} \leq c \sqrt{\tau}
$$

Proof. Since the operators $\left\{\mathcal{L}_{h} ; h \in\left[0, h_{0}\right]\right\}$ are uniformly bounded and uniformly positive definite and since the data $F_{h}$ is assumed to be uniformly bounded in $W^{1, \infty}(S ; \mathcal{Z})$, the stability estimates in Theorem 2.2 and step 1 in the proof of Theorem 2.1 in [Mie05] imply that there exists a constant $c>0$ such that

$$
\sup _{0 \leq h \leq h_{0}, 0 \leq \tau \leq T}\left\|z_{h}\right\|_{W^{1, \infty}(S ; \mathcal{Z})}+\left\|\hat{z}_{h, \tau}\right\|_{W^{1, \infty}(S ; \mathcal{Z})} \leq c
$$

Estimate (2.16) now is a consequence of Theorem 2.2 in [MPPS09].

Combining Theorem 2.3 and Theorem 2.4 we obtain an estimate for the (full) discretization error:

Corollary 2.5. Let the assumptions of Theorem 2.3 and Theorem 2.4 be satisfied. Then there exists a constant, which is independent of $\tau, t$ and $h$, such that for all $t, \tau \in S$ and $h \in\left[0, h_{0}\right]$ we have

$$
\begin{aligned}
c\left\|z(t)-\hat{z}_{h, \tau}(t)\right\|_{\mathcal{Z}} \leq \\
\quad \sqrt{\tau}+\left\|z_{0,0}-z_{0, h}\right\|_{\mathcal{Z}}+\left\|\partial_{t} z-\partial_{t} z_{h}\right\|_{L^{1}(S ; \mathcal{Z})}^{\frac{1}{2}}\left\|\left(\mathcal{L}_{h} z-F_{h}\right)-\left(\mathcal{L}_{0} z-F_{0}\right)\right\|_{L^{\infty}(S ; \mathcal{Z})}^{\frac{1}{2}} .
\end{aligned}
$$

This Corollary is the basis for deriving convergence rates of Finite Element approximations of elasto-plastic models, see Section 6. Further estimates for the term $\left(\mathcal{L}_{h}-\mathcal{L}_{0}\right) z$ rely on smoothness properties of $z$. We emphasize that Corollary2.5 is derived without any additional smoothness assumptions on the solution $z$.

\subsection{Generalized elasto-plastic models}

Let $\Omega \subset \mathbb{R}^{d}$ be a bounded domain with Lipschitz boundary, $m, n \in \mathbb{N}$. By $\operatorname{End}\left(\mathbb{R}^{s}\right)$ we denote the endomorphisms from $\mathbb{R}^{s}$ to $\mathbb{R}^{s}$. Choose $A \in L^{\infty}\left(\Omega ; \operatorname{End}\left(\mathbb{R}^{m \times d} \times \mathbb{R}^{n}\right)\right)$ and assume that $A$ is symmetric, i.e. $\left\langle A(x)\left(\begin{array}{c}F_{1} \\ z_{1}\end{array}\right),\left(\begin{array}{c}F_{2} \\ z_{2}\end{array}\right)\right\rangle=\left\langle A(x)\left(\begin{array}{c}F_{2} \\ z_{2}\end{array}\right),\left(\begin{array}{c}F_{1} \\ z_{1}\end{array}\right)\right\rangle$ for a.e. $x \in \Omega$ and 
every $F_{i} \in \mathbb{R}^{m \times d}, z_{i} \in \mathbb{R}^{n}$ and $i \in\{1,2\}$. Here, $\langle\cdot, \cdot\rangle$ stands for the scalar product in $\mathbb{R}^{m \times d} \times \mathbb{R}^{n}$. For $u \in H^{1}\left(\Omega, \mathbb{R}^{m}\right)$ and $z \in L^{2}\left(\Omega, \mathbb{R}^{n}\right)$ we define

$$
\mathcal{E}(u, z)=\frac{1}{2} \int_{\Omega}\left\langle A(x)\left(\begin{array}{c}
\nabla u(x) \\
z(x)
\end{array}\right),\left(\begin{array}{c}
\nabla u(x) \\
z(x)
\end{array}\right)\right\rangle \mathrm{d} x .
$$

Let $\mathcal{Z}=L^{2}\left(\Omega, \mathbb{R}^{n}\right)$ and assume that there exists a closed subspace $\mathcal{U} \subset H^{1}\left(\Omega, \mathbb{R}^{m}\right)$ and a constant $\alpha>0$ such that for all $u \in \mathcal{U}$ and $z \in \mathcal{Z}$ it holds

$$
\mathcal{E}(u, z) \geq \frac{\alpha}{2}\left(\|u\|_{H^{1}(\Omega)}^{2}+\|z\|_{L^{2}(\Omega)}^{2}\right) .
$$

Furthermore, let $g: \mathbb{R}^{n} \rightarrow \mathcal{P}\left(\mathbb{R}^{n}\right)$ be a maximal monotone mapping with $0 \in g(0)$. In particular, the choice $g=\partial \chi_{K}$ is admissible, where $K \subset \mathbb{R}^{n}$ is closed, convex and with $0 \in K$ and where $\chi_{K}$ denotes the characteristic function associated with $K$. We define

$$
\mathcal{G}: \mathcal{Z} \rightarrow \mathcal{P}(\mathcal{Z}), \quad \mathcal{G}(z)=\left\{\eta \in L^{2}\left(\Omega, \mathbb{R}^{n}\right) ; \eta(x) \in g(z(x)) \text { a.e. in } \Omega\right\}
$$

which is a maximal monotone mapping with respect to $\mathcal{Z}$. In this setting, Theorem 2.1 provides the existence of a unique pair $(u, z) \in W^{1,1}(S ; \mathcal{U} \times \mathcal{Z})$ satisfying (2.2) with $\mathcal{E}$ from (2.17) and $\mathcal{G}$ from (2.19). As is discussed in Section 5.1 this framework comprises elasto-plasticity with linear kinematic hardening as well as elasto-visco-plasticity including Cosserat effects.

In Sections 3-5 we study the spatial regularity properties of the solutions $(u, z)$, while in Section 6 we discuss the error due to a spatial finite element discretization and an implicit Euler scheme in time.

\section{Regularity for model problems on a cube}

\subsection{Local regularity}

The starting point of our global regularity analysis is to study the local regularity properties on cubes of solutions of the systems introduced in Section 2.3. These properties are derived with a difference quotient technique which is based on inner variations. The results in part (b) of the regularity Theorem 3.1 here below are a straightforward extension of the results from [AN09] to our slightly more general setting. In part (a) of Theorem 3.1 we discuss the local regularity properties for data which have less temporal regularity.

In the sequel we use the following notation: For matrices $T, S \in \mathbb{R}^{m \times d}$ the inner product is denoted by $S: T=\operatorname{tr}\left(T^{\top} S\right)$ with the corresponding norm $|T|=\sqrt{T: T}$. Moreover, $\mathbb{I}$ is the identity matrix in $\mathbb{R}^{d \times d}$. 
For $r>0$ let $C_{r}=(-r, r)^{d}$ be a cube with side length $2 r$. Let $m, n \in \mathbb{N}, \mathcal{U}=H_{0}^{1}\left(C_{r}, \mathbb{R}^{m}\right)$ and $\mathcal{Z}=L^{2}\left(C_{r}, \mathbb{R}^{n}\right)$. The coefficient function $A$ shall satisfy

A1 $A \in C^{0,1}\left(\overline{C_{r}}, \operatorname{End}\left(\mathbb{R}^{m \times d} \times \mathbb{R}^{n}\right)\right)$ is symmetric and there exists a constant $\alpha>0$ such that for all $u \in \mathcal{U}$ and $z \in \mathcal{Z}$ we have $\mathcal{E}(u, z) \geq \frac{\alpha}{2}\left(\|u\|_{H^{1}\left(C_{r}\right)}^{2}+\|z\|_{L^{2}\left(C_{r}\right)}^{2}\right)$.

The energy $\mathcal{E}(u, z)$ is defined in (2.17). It is assumed that the term $\ell_{1}$ in $(2.2)$ can be written as

$$
\left\langle\ell_{1}(t), v\right\rangle_{\left(\mathcal{U}^{*}, \mathcal{U}\right)}=\int_{C_{r}} f(t) \cdot v+H(t): \nabla v \mathrm{~d} x
$$

with suitable $f \in L^{\infty}\left(S ; L^{2}\left(C_{r}\right)\right)$ and $H \in L^{\infty}\left(S ; L^{2}\left(C_{r}\right)\right)$. We study the spatial regularity of functions $u \in L^{\infty}(S ; \mathcal{U})$ and $z \in W^{1,1}(S ; \mathcal{Z})$ which satisfy for a.e. $t \in S$ and every $v \in \mathcal{U}$ the relations

$$
\begin{aligned}
\int_{C_{r}}\left\langle A\left(\begin{array}{c}
\nabla u(t) \\
z(t)
\end{array}\right),\left(\begin{array}{c}
\nabla v \\
0
\end{array}\right)\right\rangle \mathrm{d} x & =\int_{C_{r}} f(t) \cdot v+H(t): \nabla v \mathrm{~d} x, \\
\partial_{t} z(t) & \in \mathcal{G}\left(-D_{z} \mathcal{E}(u(t), z(t))+\ell_{2}(t)\right) \\
z(0) & =z_{0} .
\end{aligned}
$$

In terms of the projection operators $\mathbb{P}_{m \times d}: \mathbb{R}^{m \times d} \times \mathbb{R}^{n} \rightarrow \mathbb{R}^{m \times d},(F, z) \mapsto F$ and $\mathbb{P}_{n}$ : $\mathbb{R}^{m \times d} \times \mathbb{R}^{n} \rightarrow \mathbb{R}^{n},(F, z) \mapsto z$, problem (3.2) can equivalently be written as

$$
\begin{aligned}
\int_{C_{r}} \mathbb{P}_{m \times d}\left[A\left(\begin{array}{c}
\nabla u(t) \\
z(t)
\end{array}\right)\right]: \nabla v \mathrm{~d} x & =\int_{C_{r}} f(t) \cdot v+H(t): \nabla v \mathrm{~d} x, \\
\partial_{t} z(t) & \in \mathcal{G}\left(-\mathbb{P}_{n}\left[A\left(\begin{array}{c}
\nabla u(t) \\
z(t)
\end{array}\right)\right]+\ell_{2}(t)\right) .
\end{aligned}
$$

In the sequel we use the following spaces defined for domains $\Omega_{2} \subset \Omega_{1}$ and $i \in\{1, \ldots, d\}$ :

$$
\mathcal{F}_{i}\left(\Omega_{1}, \Omega_{2}\right)=\left\{v \in L^{2}\left(\Omega_{1}\right) ; \partial_{x_{i}}\left(\left.v\right|_{\Omega_{2}}\right) \in L^{2}\left(\Omega_{2}\right)\right\}
$$

with $\|v\|_{\mathcal{F}_{i}\left(\Omega_{1}, \Omega_{2}\right)}=\|v\|_{L^{2}\left(\Omega_{1}\right)}+\left\|\partial_{x_{i}} v\right\|_{L^{2}\left(\Omega_{2}\right)}$. Moreover, finite differences are denoted by

$$
\triangle_{h e} u(t, x):=u(t, x+h e)-u(t, x)
$$

for $h \in \mathbb{R}$ and $e \in \mathbb{R}^{d} \backslash\{0\}$. By $\left\{e_{1}, \ldots, e_{d}\right\}$ we denote the standard basis in $\mathbb{R}^{d}$. The spatial regularity of solutions is discussed under different assumptions on the temporal smoothness of the data.

A2 There exists $\rho \in(0, r)$ and $i \in\{1, \ldots, d\}$ such that $z_{0} \in \mathcal{F}_{i}\left(C_{r}, C_{\rho}\right), f \in L^{\infty}\left(S ; L^{2}\left(C_{r}\right)\right)$, $H \in L^{\infty}\left(S ; \mathcal{F}_{i}\left(C_{r}, C_{\rho}\right)\right)$ and $\ell_{2} \in L^{\infty}\left(S ; \mathcal{F}_{i}\left(C_{r}, C_{\rho}\right)\right)$. 
A3 There exists $\rho \in(0, r)$ and $i \in\{1, \ldots, d\}$ such that $z_{0} \in \mathcal{F}_{i}\left(C_{r}, C_{\rho}\right), f \in W^{1,1}\left(S ; L^{2}\left(C_{r}\right)\right)$, $H \in W^{1,1}\left(S ; \mathcal{F}_{i}\left(C_{r}, C_{\rho}\right)\right)$ and $\ell_{2} \in W^{1,1}\left(S ; \mathcal{F}_{i}\left(C_{r}, C_{\rho}\right)\right)$.

Theorem 3.1 (Local regularity on cubes). Let condition A1 be satisfied.

(a) Let the pair $(u, z) \in L^{\infty}(S ; \mathcal{U}) \times W^{1,1}(S ; \mathcal{Z})$ solve (3.2) with data according to assumption A2. Then there exists $h_{0}>0$ such that

$$
\begin{gathered}
\sup _{0<h<h_{0}, t \in S} h^{-\frac{1}{2}}\left\|\triangle_{h e_{i}} \nabla u(t)\right\|_{L^{2}\left(C_{\rho / 2}\right)}<\infty \\
\sup _{0<h<h_{0}, t \in S} h^{-\frac{1}{2}}\left\|\triangle_{h e_{i}} z(t)\right\|_{L^{2}\left(C_{\rho / 2}\right)}<\infty .
\end{gathered}
$$

(b) Let the pair $(u, z) \in W^{1,1}(S ; \mathcal{U} \times \mathcal{Z})$ satisfy (3.2) with data according to A3. Then

$$
\nabla u \in L^{\infty}\left(S ; \mathcal{F}_{i}\left(C_{r}, C_{\frac{\rho}{2}}\right)\right), \quad z \in L^{\infty}\left(S ; \mathcal{F}_{i}\left(C_{r}, C_{\frac{\rho}{2}}\right)\right)
$$

If the assumptions of part (b) of Theorem 3.1 are valid for all $i \in\{1, \ldots, d\}$, then

$$
\left.u\right|_{C_{\rho / 2}} \in L^{\infty}\left(S ; H^{2}\left(C_{\rho / 2}\right)\right),\left.\quad z\right|_{C_{\rho / 2}} \in L^{\infty}\left(S ; H^{1}\left(C_{\rho / 2}\right)\right) .
$$

If the assumptions of part (a) are satisfied for every $i \in\{1, \ldots, d\}$, then it follows that

$$
\sup _{t \in S}\|u(t)\|_{B_{2, \infty}^{\frac{3}{2}}\left(C_{\rho / 2}\right)}<\infty, \quad \sup _{t \in S}\|z(t)\|_{B_{2, \infty}^{\frac{1}{2}}\left(C_{\rho / 2}\right)}<\infty .
$$

The spaces $B_{p, q}^{s}(\Omega)$ are Besov spaces and we refer to [Tri83] for a precise definition. We only recall that $v \in B_{2, \infty}^{s}(\Omega)$ for $s \in(0,1)$ if and only if $v \in L^{2}(\Omega)$ and

$$
\sup _{1 \leq i \leq d, \widetilde{\Omega} \Subset \Omega, 0<h<h_{0}} h^{-s}\left\|\triangle_{h e_{i}} v\right\|_{L^{2}(\widetilde{\Omega})}<\infty,
$$

where $\left\{e_{1}, \ldots, e_{d}\right\}$ is an arbitrary basis in $\mathbb{R}^{d}$. Moreover, for every $\delta>0$ and $s>0$ with $s \notin \mathbb{N}$ the embeddings $H^{s}(\Omega) \subset B_{2, \infty}^{s}(\Omega) \subset H^{s-\delta}(\Omega)$ are continuous. Hence, we obtain from (3.5) that for every $\delta>0$

$$
\left.u\right|_{C_{\rho / 2}} \in L^{\infty}\left(S ; H^{\frac{3}{2}-\delta}\left(C_{\rho / 2}\right)\right),\left.\quad z\right|_{C_{\rho / 2}} \in L^{\infty}\left(S ; H^{\frac{1}{2}-\delta}\left(C_{\rho / 2}\right)\right) .
$$

Proof of Theorem 3.1. Let $\rho \in(0, r)$ be given according to the assumptions in Theorem 3.1 and choose $\varphi \in C_{0}^{\infty}\left(C_{r}\right)$ with $\varphi(x)=1$ on $C_{\rho / 2}$ and $\operatorname{supp} \varphi \subset C_{\rho}$. For $h \in \mathbb{R}^{d}$ we introduce the following family of inner variations $\tau_{h}: C_{r} \rightarrow \mathbb{R}^{d}, x \rightarrow \tau_{h}(x)=x+\varphi(x) h$. 
Let $\left.h_{0}=\min \left\{\operatorname{dist}\left(\operatorname{supp} \varphi, \partial C_{r}\right),\|\varphi\|_{W^{1, \infty}\left(C_{r}\right)}\right)^{-1}\right\}$. For every $h \in \mathbb{R}^{d}$ with $|h|<h_{0}$, the mapping $\tau_{h}$ is a diffeomorphism from $C_{r}$ onto itself with $\tau_{h}(x)=x$ for every $x \in \partial C_{r}$, see e.g. [GH96]. Obviously,

$$
\nabla \tau_{h}(x)=(\mathbb{I}+h \otimes \nabla \varphi(x)), \quad \operatorname{det}\left(\nabla \tau_{h}(x)\right)=1+h \cdot \nabla \varphi(x) .
$$

Let the pair $(u, z) \in L^{\infty}(S ; \mathcal{U}) \times W^{1,1}(S ; \mathcal{Z})$ be a solution of problem (3.2) and $e_{i}$ the vector introduced in assumptions A2 and A3. For $h \in \mathbb{R} e_{i}$ with $|h|<h_{0}$ we define the shifted functions $u_{h}(t, x):=u\left(t, \tau_{h}(x)\right)$ and $z_{h}(t, x):=z\left(t, \tau_{h}(x)\right)$. Clearly, the shifted functions have the same temporal and spatial regularity as $u$ and $z$.

Straightforward calculations, which are based on a change of coordinates with $\tau_{h}$, imply that for almost every $t$ and every $v \in \mathcal{U}$ the shifted functions $u_{h}$ and $z_{h}$ satisfy

$$
\begin{aligned}
\int_{C_{r}}\left\langle A\left(\begin{array}{c}
\nabla u_{h}(t) \\
z_{h}(t)
\end{array}\right),\right. & \left.\left(\begin{array}{c}
\nabla v \\
0
\end{array}\right)\right\rangle \mathrm{d} x \\
= & \int_{C_{r}} \operatorname{det} \nabla \tau_{h} f_{h}(t) \cdot v \mathrm{~d} x+\int_{C_{r}}\left(\operatorname{det} \nabla \tau_{h}\right)\left(H_{h}(t)\left(\nabla \tau_{h}\right)^{-\top}\right): \nabla v \mathrm{~d} x \\
& +\int_{C_{r}}\left\langle F_{1}^{h}(t), \nabla v\right\rangle \mathrm{d} x \\
= & :\left\langle\ell_{1}^{h}(t), v\right\rangle_{\left(\mathcal{U}^{*}, \mathcal{U}\right) .}
\end{aligned}
$$

As before, $f_{h}(t, x)=f\left(t, \tau_{h}(x)\right), H_{h}(t, x)=H\left(t, \tau_{h}(x)\right)$ and $A_{h}=A \circ \tau_{h}$. Moreover, $F_{1}^{h}(t)$ is given by the relation

$$
\begin{aligned}
\int_{C_{r}}\left\langle F_{1}^{h}(t), \nabla v\right\rangle \mathrm{d} x= & \int_{C_{r}}\left\langle A\left(\begin{array}{c}
\nabla u_{h}(t) \\
z_{h}(t)
\end{array}\right)-\operatorname{det} \nabla \tau_{h} A_{h}\left(\begin{array}{c}
\nabla u_{h}(t)\left(\nabla \tau_{h}\right)^{-1} \\
z_{h}(t)
\end{array}\right),\left(\begin{array}{c}
\nabla v \\
0
\end{array}\right)\right\rangle \mathrm{d} x \\
& -\int_{C_{r}} \operatorname{det} \nabla \tau_{h}\left\langle A_{h}\left(\begin{array}{c}
\nabla u_{h}(t)\left(\nabla \tau_{h}\right)^{-1} \\
z_{h}(t)
\end{array}\right),\left(\begin{array}{c}
\nabla v\left(\nabla \tau_{h}^{-1}-\mathbb{I}\right) \\
0
\end{array}\right)\right\rangle \mathrm{d} x .
\end{aligned}
$$

The following evolution law is satisfied by $u_{h}$ and $z_{h}$

$$
\partial_{t} z_{h}(t) \in g\left(-D_{z} \mathcal{E}\left(u_{h}(t), z_{h}(t)\right)+F_{2}^{h}(t)+\ell_{2, h}(t)\right), \quad z_{h}(0)=z_{0} \circ \tau_{h} .
$$

Here, $\ell_{2, h}=\ell_{2} \circ \tau_{h}$ and

$$
F_{2}^{h}(t)=-\mathbb{P}_{n}\left(\left(A_{h}-A\right)\left(\begin{array}{c}
\nabla u_{h}(t) \\
z_{h}(t)
\end{array}\right)+A_{h}\left(\begin{array}{c}
\nabla u_{h}(t)\left(\nabla \tau_{h}^{-1}-\mathbb{I}\right) \\
0
\end{array}\right)\right),
$$

where $\mathbb{P}_{n}$ is the already introduced projection onto the $\mathbb{R}^{n}$ component.

From the Lipschitz continuity of the coefficient matrix $A$ and the properties of $\tau_{h}$, where we use in particular the relations in (3.6), we deduce the estimate

$$
\left\|F_{1}^{h}\right\|_{L^{\infty}\left(S ; L^{2}\left(C_{r}\right)\right)}+\left\|F_{2}^{h}\right\|_{L^{\infty}\left(S ; L^{2}\left(C_{r}\right)\right)} \leq c|h|\left(\|u\|_{L^{\infty}(S ; \mathcal{U})}+\|z\|_{L^{\infty}(S ; \mathcal{Z})}\right),
$$


and if $(u, z) \in W^{1,1}(S ; \mathcal{U} \times \mathcal{Z})$, then

$$
\left\|F_{1}^{h}\right\|_{W^{1,1}\left(S ; L^{2}\left(C_{r}\right)\right)}+\left\|F_{2}^{h}\right\|_{W^{1,1}\left(S ; L^{2}\left(C_{r}\right)\right)} \leq c|h|\left(\|u\|_{W^{1,1}(S ; \mathcal{U})}+\|z\|_{W^{1,1}(S ; \mathcal{Z})}\right) .
$$

In both inequalities, the constant $c$ is independent of $h$. By estimates (3.9) and (3.10) we have $\ell_{1}^{h} \in L^{\infty}\left(S ; \mathcal{U}^{*}\right)$ in the situation described in part (a) of Theorem 3.1 and $\ell_{1}^{h} \in$ $W^{1,1}\left(S ; \mathcal{U}^{*}\right)$ if the assumptions of part (b) are valid.

Let first the assumptions of part (a) be satisfied. From the stability estimate (2.5) applied to (3.7) and (3.8) it follows that there exists a constant $c>0$ which is independent of $h$ such that

$$
\begin{array}{r}
\left\|u-u_{h}\right\|_{L^{\infty}(S ; \mathcal{U})}^{2}+\left\|z-z_{h}\right\|_{L^{\infty}(S ; \mathcal{Z})}^{2} \leq c\left(\left\|z_{0}-z_{0, h}\right\|_{L^{2}\left(C_{r}\right)}^{2}+\left\|\ell_{1}-\ell_{1}^{h}\right\|_{L^{\infty}\left(S ; \mathcal{U}^{*}\right)}^{2}\right. \\
\left.\quad+2 c(\varphi)\|z\|_{W^{1,1}(S ; \mathcal{Z})}\left(\left\|\ell_{1}-\ell_{1}^{h}\right\|_{L^{\infty}\left(S ; \mathcal{U}^{*}\right)}+\left\|\ell_{2}-\ell_{2, h}-F_{2}^{h}\right\|_{L^{\infty}(S ; \mathcal{Z})}\right)\right) .
\end{array}
$$

In view of $\mathbf{A} \mathbf{2}$ we have (see e.g. Lemma 4.1 in [KM08])

$$
\left\|z_{0}-z_{0, h}\right\|_{L^{\infty}(S ; \mathcal{Z})} \leq c|h|\left\|z_{0}\right\|_{\mathcal{F}_{i}\left(C_{r}, C_{\rho}\right)} .
$$

The last term in (3.11) can be estimated in the same way. For estimating the terms with $\ell_{1}$ observe that

$$
\begin{aligned}
\left\|f(t)-\operatorname{det} \nabla \tau_{h} f_{h}(t)\right\|_{\mathcal{U}^{*}} & =\sup _{v \in \mathcal{U},\|v\|_{\mathcal{U}}=1} \int_{C_{r}}\left(f(t)-\operatorname{det} \nabla \tau_{h} f_{h}(t)\right) \cdot v \mathrm{~d} x \\
& =\sup _{v \in \mathcal{U},\|v\|_{\mathcal{U}}=1} \int_{C_{r}} f(t) \cdot\left(v-v \circ \tau_{h}^{-1}\right) \mathrm{d} x \leq c(\varphi)|h|\|f(t)\|_{L^{2}\left(C_{\rho}\right)} .
\end{aligned}
$$

Thus, altogether it follows that there exists a constant $\kappa>0$ such that for all $h \in \mathbb{R} e_{i} \backslash\{0\}$ with $|h|<h_{0}$ we have

$$
\begin{aligned}
& |h|^{-\frac{1}{2}}\left(\left\|\triangle_{h} u\right\|_{L^{\infty}\left(S ; H^{1}\left(C_{\rho / 2}\right)\right)}+\left\|\triangle_{h} z\right\|_{L^{\infty}\left(S ; L^{2}\left(C_{\rho / 2}\right)\right)}\right) \\
& \quad \leq \kappa\left(\left\|z_{0}\right\|_{\mathcal{F}_{i}\left(C_{r}, C_{\rho}\right)}+\|f\|_{L^{\infty}\left(S ; L^{2}\left(C_{\rho}\right)\right)}+\|H\|_{L^{\infty}\left(S ; \mathcal{F}_{i}\left(C_{r}, C_{\rho}\right)\right)}+\left\|\ell_{2}\right\|_{L^{\infty}\left(S ; \mathcal{F}_{i}\left(C_{r}, C_{\rho}\right)\right)}\right. \\
& \left.\quad+\|u\|_{L^{\infty}(S ; \mathcal{U})}+\|z\|_{W^{1,1}(S ; \mathcal{Z})}\right) .
\end{aligned}
$$

This proves the assertions of Theorem 3.1, part (a). The results in part (b) follow in the same way by applying stability estimate (2.6) to (3.7) and (3.8).

\subsection{Tangential regularity on a half cube}

For $r>0$ let $K_{r}=(-r, r)^{d-1} \times(0, r)$ be a half cube with bottom $\Gamma_{0}=(-r, r)^{d-1} \times\{0\}$ and let $m, n \in \mathbb{N}$. We choose $\mathcal{Z}=L^{2}\left(K_{r}, \mathbb{R}^{n}\right)$ and consider closed subspaces $\mathcal{U}\left(K_{r}\right) \subset$ 
$H^{1}\left(K_{r}, \mathbb{R}^{m}\right)$ allowing for different types of boundary conditions for different components of $u \in \mathcal{U}\left(K_{r}\right)$. In particular, let $D \subset\{1, \ldots, m\}, D$ might also be the empty set. Then $\mathcal{U}\left(K_{r}\right):=\left\{u \in H^{1}\left(K_{r}, \mathbb{R}^{m}\right) ;\left.u\right|_{\partial K_{r} \backslash \Gamma_{0}}=0,\left.u_{i}\right|_{\Gamma_{0}}=0\right.$ for $\left.i \in D\right\}$.

Theorem 3.2. Assume that the coefficient function A satisfies A1 from Section 3.1 with respect to $K_{r}$ and $\mathcal{U}\left(K_{r}\right) \times \mathcal{Z}$. Let the pair $(u, z) \in W^{1,1}\left(S ; \mathcal{U}\left(K_{r}\right) \times \mathcal{Z}\right)$ satisfy (3.2) on $K_{r}$ and assume that the data has the following regularity for some $\rho \in(0, r)$ :

$$
\begin{gathered}
z_{0} \in H^{1}\left(K_{r}\right), \quad f \in W^{1,1}\left(S ; L^{2}\left(K_{r}\right)\right), \quad H \in W^{1,1}\left(S ; L^{2}\left(K_{r}\right) \cap H^{1}\left(K_{\rho}\right)\right), \\
\ell_{2} \in W^{1,1}\left(S ; L^{2}\left(K_{r}\right) \cap H^{1}\left(K_{\rho}\right)\right) .
\end{gathered}
$$

Then for $1 \leq i \leq d-1$ we have the tangential regularity

$$
\partial_{x_{i}} \nabla u \in L^{\infty}\left(S ; L^{2}\left(K_{\frac{\rho}{2}}\right)\right), \quad \partial_{x_{i}} z \in L^{\infty}\left(S ; L^{2}\left(K_{\frac{\rho}{2}}\right)\right) .
$$

This theorem is a straightforward generalization of a recent result by Alber/Nesenenko [AN09], where the case $m=d$ and pure Dirichlet conditions on $\Gamma_{0}$ are considered. The theorem can be derived in the same way as the results in part (b) of Theorem 3.1 and we omit the proof. We just remark that the space $\mathcal{U}$ is invariant with respect to inner variations $\tau_{h}$ which are tangential to $\Gamma_{0}$.

\subsection{Global regularity on a half cube}

Before we formulate the key result of this paper, Theorem 3.3, we need some further notation. Let again $K_{r}=(-r, r)^{d-1} \times(0, r)$ be the half cube with bottom $\Gamma_{0}=(-r, r)^{d-1} \times$ $\{0\}$ and let $m, n \in \mathbb{N}$. By $R=\mathbb{I}-2 e_{d} \otimes e_{d}$ we denote the reflection at the boundary $\Gamma_{0}$. The extended coefficient function $A_{e}$ is defined via $A_{e}(x)=A(x)$ for $x \in \overline{K_{r}}$ and $A_{e}(x)=A(R x)$ for $x \in C_{r} \backslash K_{r}$.

Theorem 3.3 (Global regularity on a half cube). Assume that the extended coefficient function $A_{e}$ satisfies condition A1 from Section 3.1 with respect to the full cube $C_{r}$ and $H_{0}^{1}\left(C_{r}\right) \times L^{2}\left(C_{r}\right)$. Let the pair $(u, z) \in L^{\infty}\left(S ; H^{1}\left(K_{r}\right)\right) \times W^{1,1}(S ; \mathcal{Z})$ satisfy (3.2) on $K_{r}$ for all $v \in H_{0}^{1}\left(K_{r}\right)$. Assume furthermore that for all $t$ it holds $\operatorname{supp} u(t) \subset \overline{K_{\frac{3 r}{4}}}$ and that for $1 \leq i \leq d-1$ the functions $u$ and $z$ have the tangential regularity

$$
\partial_{i} \nabla u \in L^{\infty}\left(S ; L^{2}\left(K_{r}\right)\right), \partial_{i} z \in L^{\infty}\left(S ; L^{2}\left(K_{r}\right)\right) .
$$

For the data we assume that

$$
\begin{gathered}
z_{0} \in H^{1}\left(K_{r}\right), \quad f \in L^{\infty}\left(S ; L^{2}\left(K_{r}\right)\right), \\
\ell_{2}, H \in L^{\infty}\left(S ; \cap_{i=1}^{d-1} \mathcal{F}_{i}\left(K_{r}, K_{r}\right)\right) \cap L^{\infty}\left(S ; H^{1}\left(K_{\frac{r}{2}}\right)\right) .
\end{gathered}
$$


Then $\sup _{t \in S}\|u(t)\|_{B_{2, \infty}^{3 / 2}\left(K_{r / 4}\right)}<\infty, \sup _{t \in S}\|z(t)\|_{B_{2, \infty}^{1 / 2}\left(K_{r / 4}\right)}<\infty$, and for every $\delta>0$ we have

$$
u \in L^{\infty}\left(S ; H^{\frac{3}{2}-\delta}\left(K_{\frac{r}{4}}\right)\right), \quad z \in L^{\infty}\left(S ; H^{\frac{1}{2}-\delta}\left(K_{\frac{r}{4}}\right)\right) .
$$

The proof of this theorem relies on a reflection argument and is carried out in the Lemmata 3.4-3.5 here below.

Let $(u, z) \in L^{\infty}\left(S ; H^{1}\left(K_{r}\right)\right) \times W^{1,1}(S ; \times \mathcal{Z})$ be a solution to problem (3.2) on $K_{r}$ as described in Theorem 3.3. Choose a function $\varphi \in C^{\infty}([0, r])$ with $\varphi(s)=1$ in a neighborhood of $s=0, \varphi(s)=0$ for $s \geq \frac{r}{2}$ and $0 \leq \varphi \leq 1$. By $\gamma_{0}$ we denote the trace operator from $H^{1}\left(K_{r}\right)$ to $H^{\frac{1}{2}}\left(\Gamma_{0}\right)$ and define for $x=\left(x^{\prime}, x_{d}\right) \in K_{r}$

$$
\hat{u}(t, x):=\varphi\left(x_{d}\right)\left(\gamma_{0} u(t)\right)\left(x^{\prime}\right) .
$$

The tangential regularity of $u$ entails the following regularity for $\hat{u}$ :

Lemma 3.4. Under the assumptions of Theorem 3.3 it holds $\hat{u}, \partial_{d} \hat{u} \in L^{\infty}\left(S ; H^{1}\left(K_{r}\right)\right)$ with $\operatorname{supp} \hat{u}(t) \subset \overline{K_{\frac{3 r}{4}}}$.

Proof. The proof is similar to the proof of Lemma 4.2 from [Kne09] with obvious modifications.

The following extensions to $C_{r}$ will be used:

$$
u_{e}(t, x):=\left\{\begin{array}{ll}
u(t, x)-\hat{u}(t, x) & x \in K_{r} \\
-u(t, R x)+\hat{u}(t, R x) & x \in C_{r} \backslash K_{r}
\end{array} .\right.
$$

For the inner variable we use an even extension:

$$
z_{e}(t, x):= \begin{cases}z(t, x) & x \in K_{r} \\ z(t, R x) & x \in C_{r} \backslash K_{r}\end{cases}
$$

and similar for $z_{0}$, where the extension is denoted by $z_{0, e}$. The extended functions have the smoothness $u_{e} \in L^{\infty}\left(S ; H_{0}^{1}\left(C_{r}\right)\right), z_{e} \in W^{1,1}\left(S ; L^{2}\left(C_{r}\right)\right)$ and $z_{0, e} \in H^{1}\left(C_{r}\right)$. Finally let $\mathcal{E}_{e}(v, \eta)=\int_{C_{r}} \frac{1}{2}\left\langle A_{e}\left(\begin{array}{c}\nabla v \\ \eta\end{array}\right),\left(\begin{array}{c}\nabla v \\ \eta\end{array}\right)\right\rangle \mathrm{d} x$.

Lemma 3.5. Let the assumptions of Theorem 3.3 be satisfied. There exist functions $f_{e} \in$ $L^{\infty}\left(S ; L^{2}\left(C_{r}\right)\right), H_{e} \in L^{\infty}\left(S ; \mathcal{F}_{d}\left(C_{r}, C_{\frac{r}{2}}\right)\right)$ and $\ell_{2, e} \in L^{\infty}\left(S ; \mathcal{F}_{d}\left(C_{r}, C_{\frac{r}{2}}\right)\right)$ such that for all $v \in H_{0}^{1}\left(C_{r}\right)$ we have

$$
\begin{aligned}
\int_{C_{r}}\left\langle A_{e}\left(\begin{array}{c}
\nabla u_{e}(t) \\
z_{e}(t)
\end{array}\right),\left(\begin{array}{c}
\nabla v \\
0
\end{array}\right)\right\rangle \mathrm{d} x & =\int_{C_{r}} f_{e}(t) \cdot v \mathrm{~d} x+\int_{C_{r}} H_{e}(t): \nabla v \mathrm{~d} x, \\
\partial_{t} z_{e}(t) & \in \mathcal{G}\left(-D_{z} \mathcal{E}_{e}\left(u_{e}(t), z_{e}(t)\right)+\ell_{2, e}(t)\right) \\
z_{e}(0) & =z_{0, e} .
\end{aligned}
$$


Proof. Observe first that for all $v \in H_{0}^{1}\left(C_{r}\right)$ it holds with $\widetilde{v}(x)=v(R x)$

$$
\begin{aligned}
\int_{C_{r}}\left\langle A_{e}\left(\begin{array}{c}
\nabla u_{e} \\
z_{e}
\end{array}\right),\left(\begin{array}{c}
\nabla v \\
0
\end{array}\right)\right\rangle \mathrm{d} x= & \int_{K_{r}}\left\langle A\left(\begin{array}{c}
\nabla u \\
z
\end{array}\right),\left(\begin{array}{c}
\nabla(v-\widetilde{v}) \\
0
\end{array}\right)\right\rangle \mathrm{d} x-\int_{K_{r}}\left\langle A\left(\begin{array}{c}
\nabla \hat{u} \\
0
\end{array}\right),\left(\begin{array}{c}
\nabla v \\
0
\end{array}\right)\right\rangle \mathrm{d} x \\
& +\int_{C_{r} \backslash K_{r}}\left\langle\left. A_{e}\left(\begin{array}{c}
\nabla u \\
z
\end{array}\right)\right|_{R x},\left(\begin{array}{c}
\nabla v(R+\mathbb{I}) \\
0
\end{array}\right)\right\rangle \mathrm{d} x \\
& +\int_{C_{r} \backslash K_{r}}\left\langle\left. A_{e}\left(\begin{array}{c}
\nabla \hat{u} R-\nabla u(R+\mathbb{I}) \\
0
\end{array}\right)\right|_{R x},\left(\begin{array}{c}
\nabla v \\
0
\end{array}\right)\right\rangle \mathrm{d} x .
\end{aligned}
$$

Since the pair $(u, z)$ solves (3.3) and since $v-\widetilde{v} \in H_{0}^{1}\left(K_{r}\right)$, we may replace the first term on the right hand side with $f$ and $H$ and obtain after rearranging the terms the following relation:

$$
\begin{aligned}
\int_{C_{r}}\left\langle A_{e}\left(\begin{array}{c}
\nabla u_{e} \\
z_{e}
\end{array}\right),\left(\begin{array}{c}
\nabla v \\
0
\end{array}\right)\right\rangle \mathrm{d} x & =\int_{K_{r}} f v \mathrm{~d} x+\int_{C_{R} \backslash K_{r}}(-f \circ R) v \mathrm{~d} x \\
& -\int_{C_{r} \backslash K_{r}} H \circ R(R+\mathbb{I}): \nabla v \mathrm{~d} x \\
& +\int_{C_{r} \backslash K_{r}}\left(\mathbb{P}_{m \times d}\left[A\left(\begin{array}{c}
\nabla u \\
z
\end{array}\right)\right] \circ R\right)(R+\mathbb{I}): \nabla v \mathrm{~d} x \\
& +\int_{K_{r}} H: \nabla v \mathrm{~d} x+\int_{C_{r} \backslash K_{r}} H \circ R: \nabla v \mathrm{~d} x \\
& -\int_{K_{r}} \mathbb{P}_{m \times d}\left[A\left(\begin{array}{c}
\nabla \hat{u} \\
0
\end{array}\right)\right]: \nabla v \mathrm{~d} x \\
& +\int_{C_{r} \backslash K_{r}} \mathbb{P}_{m \times d}\left[A\left(\begin{array}{c}
\nabla \hat{u} R-\nabla u(R+\mathbb{I}) \\
0
\end{array}\right)\right] \circ R: \nabla v \mathrm{~d} x .
\end{aligned}
$$

Observe that the regularity assumption on $u$ and $z$ imply that

$$
\operatorname{div}\left(\left(H \circ R-\mathbb{P}_{m \times d}\left[A\left(\begin{array}{c}
\nabla u \\
z
\end{array}\right)\right] \circ R\right)(R+\mathbb{I})\right) \in L^{\infty}\left(S ; L^{2}\left(C_{r} \backslash K_{r}\right)\right)
$$

since due to the factor $R+\mathbb{I}$ the derivative with respect to $x_{d}$ does not appear. Thus, after applying the Gauss Theorem, the first four integrals on the right hand side in (3.19) can be replaced with the term $\int_{C_{r}} f_{e}(t, x) \cdot v(x) \mathrm{d} x$, where

$$
f_{e}(t, x)= \begin{cases}f(t, x) & x \in K_{r}, \\
-f(t, R x)+2 \operatorname{div}_{x^{\prime}}\left(H(t, R x)-\mathbb{P}_{m \times d}\left[A\left(\begin{array}{c}
\nabla u(t) \\
z(t)
\end{array}\right)\right] \circ R\right) & x \in C_{r} \backslash K_{r} .\end{cases}
$$

Here, we use the notation $\operatorname{div}_{x^{\prime}} \sigma=\partial_{x_{1}} \sigma_{1}+\ldots+\partial_{x_{d-1}} \sigma_{d-1}=\frac{1}{2} \operatorname{div}(\sigma(R+\mathbb{I}))$ for $\sigma: C_{r} \rightarrow$ $\mathbb{R}^{m \times d}$, and $\sigma_{i}$ is the $i$-th column of $\sigma$. Note that $f_{e} \in L^{\infty}\left(S ; L^{2}\left(C_{r}\right)\right)$. 
Let

$$
\theta_{e}(t, x)= \begin{cases}\nabla \hat{u}(t, x) & x \in K_{r}, \\ -\nabla(\hat{u}(t, R x))+(\nabla(u(t, R x)))(R+\mathbb{I}) & x \in C_{r} \backslash K_{r} .\end{cases}
$$

From the assumptions on $u$ and from Lemma 3.4 we conclude that $\partial_{d}\left(\left.\theta_{e}\right|_{K_{r}}\right) \in L^{\infty}\left(S ; L^{2}\left(K_{r}\right)\right)$ and $\left.\partial_{d} \theta_{e}\right|_{C_{r} \backslash K_{r}} \in L^{\infty}\left(S ; L^{2}\left(C_{r} \backslash K_{r}\right)\right)$. Since the traces on $\Gamma_{0}$ of $\left.\theta_{e}\right|_{K_{r}}$ and of $\left.\theta_{e}\right|_{C_{r} \backslash K_{r}}$ coincide, it follows that $\theta_{e} \in L^{\infty}\left(S ; \mathcal{F}_{d}\left(C_{r}, C_{r}\right)\right)$. Moreover, we define

$$
H_{e}(t, x)=-\mathbb{P}_{m \times d}\left[A_{e}(x)\left(\begin{array}{cl}
\theta_{e}(t, x) \\
0
\end{array}\right)\right]+ \begin{cases}H(t, x) & x \in K_{r}, \\
H(t, R x) & x \in C_{r} \backslash K_{r} .\end{cases}
$$

The assumptions on $H$ and the properties of $\theta_{e}$ imply that $H_{e} \in L^{\infty}\left(S ; \mathcal{F}_{d}\left(C_{r}, C_{\frac{r}{2}}\right)\right)$. With these definitions, the right hand side in (3.19) is equal to $\int_{C_{r}} f_{e}(t) \cdot v \mathrm{~d} x+\int_{C_{r}} H_{e}(t): \nabla v \mathrm{~d} x$, which leads to the first equation in (3.18).

Finally we define

$$
\ell_{2, e}(t, x)=-\mathbb{P}_{n}\left[A_{e}(x)\left(\begin{array}{cl}
\theta_{e}(t, x) \\
0
\end{array}\right)\right]+ \begin{cases}\ell_{2}(t, x) & x \in K_{r}, \\
\ell_{2}(t, R x) & x \in C_{r} \backslash K_{r} .\end{cases}
$$

As before we have $\ell_{2, e} \in L^{\infty}\left(S ; \mathcal{F}_{d}\left(C_{r}, C_{\frac{r}{2}}\right)\right)$. Moreover, straightforward calculations show that the extended functions satisfy the second relation in (3.18). This finishes the proof of Lemma 3.5.

Proof of Theorem 3.3. Theorem 3.3 is an immediate consequence of part (a) of Theorem 3.1 and of Lemma 3.5.

Observe that even with stronger assumptions on the temporal regularity of the data we cannot extend in the proof of Theorems 3.1 and 3.2 the regularity of $u$ from $L^{\infty}\left(S ; H_{\text {loc }}^{2}\left(K_{r}\right) \cap\right.$ $\left.H_{\text {tang }}^{2}\left(K_{r}\right)\right)$ to $W^{1,1}\left(S ; H_{\text {loc }}^{2}\left(K_{r}\right) \cap H_{\text {tang }}^{2}\left(K_{r}\right)\right)$. In fact, the example in Section 5.2.4 shows that in spite of arbitrary smooth data, $u$ does not belong to $W^{1,1}\left(S ; H^{2}(\Omega)\right)$ in general. Thus we cannot expect that the extended data in the proof of Theorem 3.3 (see Lemma $3.5)$, which contain tangential derivatives of $u$ and $z$, have the temporal regularity formulated in assumption A3. Hence, in order to obtain the global regularity, we can only apply the weak result formulated in part (a) of Theorem 3.1, and not the stronger result stated in part (b) of Theorem 3.1. This explains the loss of a "half" derivative in the normal direction. However, for time independent problems, where the model reduces to a linear elliptic system of partial differential equations, the reflection argument gives a full additional spatial derivative. 


\section{Main regularity theorem}

\subsection{Basic assumptions and main result}

We are now ready to formulate and prove the main regularity theorem for generalized elasto-(visco)-plastic models on smooth domains. In particular we assume the following:

$\mathbf{R} 1 \Omega \subset \mathbb{R}^{d}$ is a bounded domain with $C^{1,1}$-smooth boundary, see e.g. [Gri85].

For $A \in L^{\infty}\left(\Omega, \operatorname{End}\left(\mathbb{R}^{m \times d} \times \mathbb{R}^{n}\right)\right), u \in H^{1}\left(\Omega, \mathbb{R}^{m}\right)$ and $z \in L^{2}\left(\Omega, \mathbb{R}^{n}\right)$ the energy $\mathcal{E}$ is defined as in (2.17):

$$
\mathcal{E}(u, z)=\frac{1}{2} \int_{\Omega}\left\langle A\left(\begin{array}{c}
\nabla u \\
z
\end{array}\right),\left(\begin{array}{c}
\nabla u \\
z
\end{array}\right)\right\rangle \mathrm{d} x
$$

R2 The coefficient function $A$ belongs to $C^{0,1}\left(\bar{\Omega}, \operatorname{End}\left(\mathbb{R}^{m \times d} \times \mathbb{R}^{n}\right)\right)$, is self adjoint and the energy satisfies $\mathcal{E}(v, z) \geq \frac{\alpha}{2}\left(\|v\|_{H^{1}(\Omega)}^{2}+\|z\|_{L^{2}(\Omega)}^{2}\right)$ for all $v \in H_{0}^{1}(\Omega)$ and $z \in L^{2}(\Omega)$.

Note that $\mathbf{R} 2$ shall be satisfied for $v \in H_{0}^{1}(\Omega)$, only, independently of the type of boundary conditions which finally are imposed on the generalized displacements.

R3 $g: \mathbb{R}^{n} \rightarrow \mathcal{P}\left(\mathbb{R}^{n}\right)$ is maximal monotone with $0 \in g(0)$. Moreover, $\mathcal{G}: L^{2}\left(\Omega, \mathbb{R}^{n}\right) \rightarrow$ $\mathcal{P}\left(L^{2}\left(\Omega, \mathbb{R}^{n}\right)\right)$ is defined as in $(2.19)$.

$\mathbf{R} 4 z_{0} \in H^{1}\left(\Omega, \mathbb{R}^{n}\right), f \in W^{1,1}\left(S ; L^{2}\left(\Omega, \mathbb{R}^{m}\right)\right), H \in W^{1,1}\left(S ; H^{1}\left(\Omega, \mathbb{R}^{m \times d}\right)\right)$, $\ell_{2} \in W^{1,1}\left(S ; H^{1}\left(\Omega, \mathbb{R}^{n}\right)\right)$ and $u_{0} \in W^{1,1}\left(S ; H^{\frac{3}{2}}\left(\partial \Omega, \mathbb{R}^{m}\right)\right)$.

For $D \subset\{1, \ldots, m\}$, where $D=\emptyset$ is not excluded, the set of admissible generalized displacements is given by

$$
\mathcal{U}=\left\{v \in H^{1}\left(\Omega, \mathbb{R}^{m}\right) ;\left.v_{i}\right|_{\partial \Omega}=0 \text { for } i \in D\right\} .
$$

With this choice it is possible to define different types of boundary conditions for the different components of $u$.

We consider functions $(u, z) \in W^{1,1}\left(S ; H^{1}\left(\Omega, \mathbb{R}^{m}\right)\right) \times W^{1,1}\left(S ; L^{2}\left(\Omega, \mathbb{R}^{n}\right)\right)$ which for all $v \in \mathcal{U}$ and a.e. $t \in S$ satisfy the following relations

$$
\begin{aligned}
D_{u} \mathcal{E}(u(t), z(t))[v] & =\int_{\Omega}\left\langle A\left(\begin{array}{c}
\nabla u(t) \\
z(t)
\end{array}\right),\left(\begin{array}{c}
\nabla v \\
0
\end{array}\right)\right\rangle \mathrm{d} x=\int_{\Omega} f(t) \cdot v+H(t): \nabla v \mathrm{~d} x, \\
\partial_{t} z(t) & \in \mathcal{G}\left(-D_{z} \mathcal{E}(t, u(t), z(t))+\ell_{2}(t)\right), \\
z(0) & =z_{0}, \\
\left.u_{i}(t)\right|_{\partial \Omega} & =u_{0, i}(t) \text { for } i \in D .
\end{aligned}
$$


Theorem 4.1 (Main Regularity Theorem). Let $\mathbf{R} \mathbf{1}-\mathbf{R} 4$ be satisfied and assume that the pair $(u, z) \in W^{1,1}\left(S ; H^{1}(\Omega)\right) \times W^{1,1}\left(S ; L^{2}(\Omega)\right)$ satisfies $(4.2)$ for all $v \in \mathcal{U}$ and almost every $t \in S$. Then

$$
\sup _{t \in S}\|u(t)\|_{B_{2, \infty}^{\frac{3}{2}}(\Omega)}<\infty, \quad \sup _{t \in S}\|z(t)\|_{B_{2, \infty}^{\frac{1}{2}}(\Omega)}<\infty
$$

and for every $\delta>0$ we have

$$
u \in L^{\infty}\left(S ; H^{\frac{3}{2}-\delta}(\Omega)\right), \quad z \in L^{\infty}\left(S ; H^{\frac{1}{2}-\delta}(\Omega)\right) .
$$

The optimality of Theorem 4.3 and further examples are discussed in Section 5. The proof of Theorem 4.1 is carried out in Sections 4.2-4.3. By the usual arguments we may assume for the Dirichlet datum that $u_{0} \equiv 0$ and thus $u \in W^{1,1}(S ; \mathcal{U})$.

\subsection{Step 1: Localization of the model and tangential regularity}

Assumption R1 implies that for every $y_{0} \in \partial \Omega$ there exists a neighborhood $V_{y_{0}}$ of $y_{0}$ and a $C^{1,1}$-diffeomorphism $\Phi_{y_{0}}: V_{y_{0}} \rightarrow C_{1}$ having the properties $\Phi_{y_{0}}\left(y_{0}\right)=0, \Phi_{y_{0}}\left(\partial \Omega \cap V_{y_{0}}\right)=\Gamma_{0}$, $\Phi_{y_{0}}\left(\Omega \cap V_{y_{0}}\right)=K_{1}$ and $\Phi_{y_{0}}\left(V_{y_{0}} \backslash \bar{\Omega}\right)=C_{1} \backslash K_{1}$. The diffeomorphism $\Phi_{y_{0}}$ is chosen in such a way that $\operatorname{det} \nabla \Phi_{y_{0}}$ is constant. This choice is always possible for $C^{1,1}$-smooth domains, see for example [Gri85]. The inverse of $\Phi_{y_{0}}$ is denoted by $\Psi_{y_{0}}: C_{1} \rightarrow V_{y_{0}}$.

Let $A \in C^{0,1}\left(\bar{\Omega} ; \operatorname{End}\left(\mathbb{R}^{m \times d} \times \mathbb{R}^{n}\right)\right)$ be the coefficient function in (4.2). For $x \in \overline{K_{1}}$, $F_{i} \in \mathbb{R}^{m \times d}, z_{i} \in \mathbb{R}^{n}$ we define $A_{\Phi_{y_{0}}} \in C^{0,1}\left(\overline{K_{1}} ; \operatorname{End}\left(\mathbb{R}^{m \times d} \times \mathbb{R}^{n}\right)\right)$ via

$$
\left\langle A_{\Phi_{y_{0}}}(x)\left(\begin{array}{c}
F_{1} \\
z_{1}
\end{array}\right),\left(\begin{array}{c}
F_{2} \\
z_{2}
\end{array}\right)\right\rangle=\left\langle A\left(\Psi_{y_{0}}(x)\right)\left(\underset{F_{1}}{F_{1}\left(\nabla \Psi_{y_{0}}(x)\right)^{-1}}\right),\left(\underset{F_{2}\left(\nabla \Psi_{y_{2}}(x)\right)^{-1}}{z_{2}}\right)\right\rangle
$$

Moreover,

$$
\mathcal{E}_{\Phi_{y_{0}}}(v, \zeta):=\frac{1}{2} \int_{K_{1}}\left\langle A_{\Phi_{y_{0}}}\left(\begin{array}{c}
\nabla v \\
\zeta
\end{array}\right),\left(\begin{array}{c}
\nabla_{\zeta} v \\
\zeta
\end{array}\right)\right\rangle \mathrm{d} x
$$

Finally let $\mathcal{W}\left(K_{r}\right)=\left\{v \in H^{1}\left(K_{r}\right) ;\left.v\right|_{\partial K_{r} \backslash \Gamma_{0}}=0\right\}$ for $r>0$.

Lemma 4.2. Let conditions $\mathbf{R} 1$ and $\mathbf{R} 2$ be satisfied. For every $y_{0} \in \partial \Omega$ there exists $r \in(0,1)$ such that for all $v \in \mathcal{W}\left(K_{r}\right)$ and $\zeta \in L^{2}\left(K_{r}\right)$ it holds

$$
\mathcal{E}_{\Phi_{y_{0}}}(v, \zeta) \geq \frac{\alpha}{4}\left(\|\nabla v\|_{L^{2}\left(K_{r}\right)}^{2}+\|\zeta\|_{L^{2}\left(K_{r}\right)}^{2}\right)
$$

with $\alpha$ from $\mathbf{R 2}$.

Proof. Assume that conditions R1 and R2 are satisfied. By a localization argument similar to the one described in [GH96, Chap. 4.1.3, Legendre-Hadamard condition] and a scaling argument it follows that for all $x_{0} \in \bar{\Omega}, r>0, v \in H_{0}^{1}\left(C_{r}\right)$ and $\zeta \in L^{2}\left(C_{r}\right)$ it holds

$$
\int_{C_{r}}\left\langle A\left(x_{0}\right)\left(\begin{array}{c}
\nabla v \\
\zeta
\end{array}\right),\left(\begin{array}{c}
\nabla v \\
\zeta
\end{array}\right)\right\rangle \mathrm{d} x \geq \alpha\left(\|\nabla v\|_{L^{2}\left(C_{r}\right)}^{2}+\|\zeta\|_{L^{2}\left(C_{r}\right)}^{2}\right) .
$$


Here, $\alpha$ is the same constant as in condition $\mathbf{R} 2$ and does not depend on $r$. Moreover, by using even extensions for $v \in \mathcal{W}\left(K_{r}\right)$ and odd extensions for $\zeta \in L^{2}\left(K_{r}\right)$ from $K_{r}$ to $C_{r}$, it follows that estimate $(4.5)$ is valid also on $\mathcal{W}\left(K_{r}\right) \times L^{2}\left(K_{r}\right)$ with the same constant $\alpha$ as in (4.5).

Let now $y_{0} \in \partial \Omega$ be arbitrary. For all $r \in(0,1], v \in \mathcal{W}\left(K_{r}\right)$ and $\zeta \in L^{2}\left(K_{r}\right)$ we have

$$
\begin{aligned}
2 \mathcal{E}_{\Phi_{y_{0}}}(v, \zeta) & =\int_{K_{r}}\left\langle A\left(y_{0}\right)\left(\begin{array}{c}
\nabla v \\
\zeta
\end{array}\right),\left(\begin{array}{c}
\nabla v \\
\zeta
\end{array}\right)\right\rangle \mathrm{d} x+\int_{K_{r}}\left\langle\left(A_{\Phi_{y_{0}}}(x)-A\left(y_{0}\right)\right)\left(\begin{array}{c}
\nabla v \\
\zeta
\end{array}\right),\left(\begin{array}{c}
\nabla v \\
\zeta
\end{array}\right)\right\rangle \mathrm{d} x \\
& \geq\left(\alpha-c_{A, \Phi_{y_{0}}} \operatorname{diam}\left(K_{r}\right)\right)\left(\|\nabla v\|_{L^{2}\left(C_{r}\right)}^{2}+\|\zeta\|_{L^{2}\left(C_{r}\right)}^{2}\right) .
\end{aligned}
$$

The constant $c_{A, \Phi_{y_{0}}}$ depends on $\Phi_{y_{0}}$ and on the Lipschitz properties of $A$, but is independent of $r$. For small enough $r$ we therefore arrive at (4.4).

In the sequel we omit the index $y_{0}$.

Let $(u, z) \in W^{1,1}(S ; \mathcal{U}) \times W^{1,1}\left(S ; L^{2}(\Omega)\right)$ be given as in Theorem 4.1, choose $y_{0} \in \partial \Omega$ and let $r \in(0,1)$ be given according to Lemma 4.2. Let furthermore $\varphi \in C_{0}^{\infty}\left(C_{\frac{3 r}{4}}\right)$ with $0 \leq \varphi \leq 1$ and with $\varphi \equiv 1$ on $C_{\frac{r}{2}}$. For $(t, x) \in S \times K_{r}$ we define

$$
u_{\Phi}(t, x)=\varphi(x) u(t, \Psi(x)), \quad z_{\Phi}(t, x)=z(t, \Psi(x)) .
$$

Furthermore, the space

$$
\mathcal{U}\left(K_{r}\right)=\left\{w \in H^{1}\left(K_{r}\right) ;\left.v\right|_{\partial K_{r} \backslash \Gamma_{0}}=0,\left.v_{i}\right|_{\Gamma_{0}}=0 \text { for } i \in D\right\}
$$

is defined in the same way as in Section 3.2. Obviously, $\mathcal{U}\left(K_{r}\right) \subset \mathcal{W}\left(K_{r}\right)$ and

$$
\left(u_{\Phi}, z_{\Phi}\right) \in W^{1,1}\left(S ; \mathcal{U}\left(K_{r}\right)\right) \times W^{1,1}\left(S ; L^{2}\left(K_{r}\right)\right) .
$$

Testing (4.2) with $v \circ \Phi$, where $v \in \mathcal{U}\left(K_{r}\right)$, changing the coordinates using $\Phi$ and moving the lower order terms to the right hand side, we arrive at the following relations taking into account that $|\operatorname{det} \nabla \Phi|$ is constant:

For all $v \in \mathcal{U}\left(K_{r}\right)$ and almost every $t \in S$ it holds

$$
\begin{aligned}
\int_{K_{r}}\left\langle A_{\Phi}(x)\left(\begin{array}{c}
\nabla u_{\Phi}(t) \\
z_{\Phi}(t)
\end{array}\right),\left(\begin{array}{c}
\nabla v \\
0
\end{array}\right)\right\rangle \mathrm{d} x & =\int_{K_{r}} f_{\Phi}(t) \cdot v \mathrm{~d} x+H_{\Phi}(t): \nabla v \mathrm{~d} x, \\
\partial_{t} z_{\Phi}(t) & \in \mathcal{G}\left(-\mathbb{P}_{n}\left[A_{\Phi}\left(\begin{array}{c}
\nabla u_{\Phi}(t) \\
z_{\Phi}(t)
\end{array}\right)\right]+\ell_{2, \Phi}(t)\right), \\
z_{\Phi}(0) & =z_{0, \Phi} .
\end{aligned}
$$

Here, $z_{0, \Phi}=z_{0} \circ \Psi$. Moreover, with $\widetilde{u}(t, x)=u(t, \Psi(x))$ we have

$$
\begin{aligned}
f_{\Phi}(t) & =f(t) \circ \Psi \\
H_{\Phi}(t) & =H(t) \circ \Psi \nabla \Psi^{-\top}-\mathbb{P}_{m \times d}\left[A_{\Phi}\left(\begin{array}{c}
\nabla((-\varphi) \widetilde{u}) \\
0
\end{array}\right)\right], \\
\ell_{2, \Phi}(t) & =\ell_{2}(t) \circ \Psi-\mathbb{P}_{n}\left[A_{\Phi}\left(\begin{array}{c}
\nabla((1-\varphi) \widetilde{u}) \\
0
\end{array}\right)\right] .
\end{aligned}
$$


From assumption $\mathbf{R} 4$ and using that $(1-\varphi) \widetilde{u}=0$ on $K_{r / 2}$, we obtain

$$
\begin{gathered}
f_{\Phi} \in W^{1,1}\left(S ; L^{2}\left(K_{r}\right)\right), \quad H_{\Phi} \in W^{1,1}\left(S ; L^{2}\left(K_{r}\right)\right) \cap W^{1,1}\left(S ; H^{1}\left(K_{\frac{r}{2}}\right)\right), \\
\ell_{2, \Phi} \in W^{1,1}\left(S ; L^{2}\left(K_{r}\right)\right) \cap W^{1,1}\left(S ; H^{1}\left(K_{\frac{r}{2}}\right)\right) .
\end{gathered}
$$

In view of Lemma 4.2 we are now exactly in the situation described in Section 3.2 on tangential regularity. Theorem 3.2 therefore implies that for $1 \leq i \leq d-1$ we have

$$
\partial_{x_{i}} \nabla u_{\Phi} \in L^{\infty}\left(S ; L^{2}\left(K_{\frac{r}{4}}\right)\right), \quad \partial_{x_{i}} z_{\Phi} \in L^{\infty}\left(S ; L^{2}\left(K_{\frac{r}{4}}\right)\right) .
$$

Since $y_{0} \in \partial \Omega$ was arbitrary and since $\partial \Omega$ can be covered with a finite number of the domains $\Psi_{y_{0}}\left(K_{\frac{r}{4}}\right)$, the tangential regularity result is also valid for $u$ and $z$ on the whole domain $\Omega$.

\subsection{Step 2: Global regularity}

We consider again the localized problem (4.6). Thanks to the first step we have the additional regularity $\partial_{i} \nabla u_{\Phi} \in L^{\infty}\left(S ; L^{2}\left(K_{r}\right)\right)$ and $\partial_{i} z_{\Phi} \in L^{\infty}\left(S ; L^{2}\left(K_{r}\right)\right)$ for $1 \leq i \leq d-1$. Thus, in addition to (4.8) the data in (4.7) satisfy

$$
H_{\Phi}, \ell_{2, \Phi} \in L^{\infty}\left(S ; \cap_{i=1}^{d-1} \mathcal{F}_{i}\left(K_{r}, K_{r}\right)\right) .
$$

By a reflection argument it follows from Lemma 4.2 that the extended coefficient function $A_{\Phi, e}$, which is defined by $A_{\Phi, e}(x)=A_{\Phi}(x)$ for $x \in K_{r}$ and $A_{\Phi, e}(x)=A_{\Phi}(R x)$ for $x \in C_{r} \backslash K_{r}$, satisfies

$$
\int_{C_{r}}\left\langle A_{\Phi, e}\left(\begin{array}{c}
\nabla v \\
\zeta
\end{array}\right),\left(\begin{array}{c}
\nabla v \\
\zeta
\end{array}\right)\right\rangle \mathrm{d} x \geq \kappa\left(\|v\|_{H^{1}\left(C_{r}\right)}^{2}+\|\zeta\|_{L^{2}\left(C_{r}\right)}^{2}\right)
$$

for all $v \in H_{0}^{1}\left(C_{r}\right), \zeta \in L^{2}\left(C_{r}\right)$ and some constant $\kappa>0$. Theorem 3.3 now guarantees that $\sup _{t \in S}\left\|u_{\Phi}(t)\right\|_{B_{2, \infty}^{3 / 2}\left(K_{r / 4}\right)}<\infty, \sup _{t \in S}\left\|z_{\Phi}(t)\right\|_{B_{2, \infty}^{1 / 2}\left(K_{r / 4}\right)}<\infty$, and that for every $\delta>0$ we have $u_{\Phi} \in L^{\infty}\left(S ; H^{\frac{3}{2}-\delta}\left(K_{\frac{r}{4}}\right)\right)$ and $z_{\Phi} \in L^{\infty}\left(S ; H^{\frac{1}{2}-\delta}\left(K_{\frac{r}{4}}\right)\right)$. Since $y_{0} \in \partial \Omega$ is arbitrary and since $\partial \Omega$ can be covered with a finite number of domains $\Psi_{y_{0}}\left(K_{\frac{r}{4}}\right)$, we arrive finally at (4.3) and the proof of Theorem 4.1 is finished.

\section{Examples and Discussion}

\subsection{Application to elasto-visco-plastic models}

The Regularity Theorem 4.1 is in particular applicable to classical elastic-plastic models with linear hardening having positive definite hardening coefficients and to elastic-plastic models with Cosserat-effects. 


\subsubsection{Elasto-(visco)-plasticity with linear hardening}

For elasto-plastic models with linear hardening we have $m=d$. Let $\varepsilon: \mathbb{R}^{d \times d} \rightarrow \mathbb{R}_{\text {sym }}^{d \times d}$ denote the linearized strain tensor, which is defined through $\varepsilon(F)=\frac{1}{2}\left(F+F^{\top}\right)$ for $F \in \mathbb{R}^{d \times d}$. Let furthermore $C \in C^{1,1}\left(\Omega, \operatorname{End}\left(\mathbb{R}_{\mathrm{sym}}^{d \times d}\right)\right)$. The tensor $C$ corresponds to the elasticity tensor. It is assumed that $C$ is self adjoint and that there exists a constant $\alpha>0$ such that $C(x) F: F \geq \alpha|F|^{2}$ for all $F \in \mathbb{R}_{\mathrm{sym}}^{d \times d}$ and all $x \in \Omega$. Let moreover $L \in C^{1,1}\left(\Omega ; \operatorname{End}\left(\mathbb{R}^{n}\right)\right)$ be self adjoint and uniformly positive definite and choose $B \in C^{1,1}\left(\Omega, \operatorname{Lin}\left(\mathbb{R}^{n}, \mathbb{R}_{\mathrm{sym}}^{d \times d}\right)\right)$. $B$ maps the vector $z$ of internal variables onto the plastic strain. For $u \in H^{1}\left(\Omega, \mathbb{R}^{d}\right)$ and $z \in L^{2}\left(\Omega, \mathbb{R}^{n}\right)$ the stored energy reads

$$
\mathcal{E}_{\mathrm{H}}(u, z)=\frac{1}{2} \int_{\Omega} C(\varepsilon(\nabla u)-B z):(\varepsilon(\nabla u)-B z)+(L z) \cdot z \mathrm{~d} x .
$$

Since $C$ and $L$ are assumed to be positive definite, it follows with Korn's inequality that estimate (2.18) is satisfied for all $u \in H_{0}^{1}(\Omega)$ and $z \in \mathcal{Z}$. Problem (2.2) formulated with $\mathcal{E}_{\mathrm{H}}$ from (5.1) and $\mathcal{G}$ from (2.19) constitutes an elastic-(visco)-plastic model with linear hardening and takes the form: Find $(u, z) \in W^{1,1}(S ; \mathcal{U}) \times W^{1,1}(S ; \mathcal{Z})$ such that for a.e. $t \in S$ and every $v \in \mathcal{U}$

$$
\begin{array}{r}
\int_{\Omega} C(\varepsilon(\nabla u(t))-B z(t)): \varepsilon(\nabla v) \mathrm{d} x=\left\langle\ell_{1}(t), v\right\rangle_{\left(\mathcal{U}^{*}, \mathcal{U}\right)}, \\
\partial_{t} z(t) \in g\left(-\left(-B^{\top} C(\varepsilon(\nabla u(t))-B z(t))+L z(t)\right)+\ell_{2}(t)\right) .
\end{array}
$$

The global regularity theorem 4.1 is applicable to this model. Observe that $g$ can be chosen according to the von Mises flow rule as well as according to the Tresca flow rule.

This setting comprises linear kinematic hardening while pure isotropic hardening is excluded in our analysis. In the pure isotropic case, the matrix $L$ is positive semidefinite, only. We refer to [HR99, Joh78] for an existence proof for the case with isotropic hardening. Hölder regularity of solutions, also in the isotropic case, is investigated in [FL08a].

\subsubsection{Elasto-plasticity coupled with Cosserat micropolar effects}

In [NC05] an elastic-plastic model was introduced which incorporates Cosserat micropolar effects. This model is analyzed in [NC05, NC08] with respect to existence and local regularity and in [KN08] with respect to global regularity of a time discretized version. Let $\Omega \subset \mathbb{R}^{d}, d=3$, be a bounded domain. In this model, not only the displacements $u$ but also linearized micro-rotations $Q$ are taken into account. These micro-rotations are represented with skew-symmetric tensors which are identified with vectors in $\mathbb{R}^{\frac{d(d-1)}{2}}$. Consequently, $m=d+d(d-1) / 2$. The generalized displacements are now given by the pair 
$(u, Q) \in \mathbb{R}^{d} \times \mathbb{R}_{\text {skew }}^{d \times d} \cong \mathbb{R}^{m}$. The internal variable $z$ is identified with the plastic strain tensor $z=\varepsilon_{p} \in \mathbb{R}_{\text {sym, dev }}^{d \times d} \cong \mathbb{R}^{n}$ with a suitable $n \in \mathbb{N}$. The set $\mathbb{R}_{\text {sym, dev }}^{d \times d}$ consists of the symmetric matrices with zero trace. For $u \in H^{1}\left(\Omega, \mathbb{R}^{d}\right), Q \in H^{1}\left(\Omega, \mathbb{R}_{\text {skew }}^{d \times d}\right)$ and $\varepsilon_{p} \in L^{2}\left(\Omega, \mathbb{R}_{\text {sym,dev }}^{d \times d}\right)$ the stored energy reads

$$
\mathcal{E}_{\mathrm{C}}\left((u, Q), \varepsilon_{p}\right)=\int_{\Omega} \mu\left|\varepsilon(\nabla u)-\varepsilon_{p}\right|^{2}+\mu_{c} \mid \text { skew }\left.(\nabla u-Q)\right|^{2}+\frac{\lambda}{2}|\operatorname{tr} \nabla u|^{2}+\gamma|\nabla Q|^{2} \mathrm{~d} x .
$$

Here, $\lambda, \mu>0$ are the Lamé constants, $\mu_{c}>0$ is the Cosserat couple modulus and $\gamma>0$ depends on the Lamé constants and an internal length parameter. Let $\mathcal{U}=H_{0}^{1}\left(\Omega, \mathbb{R}^{d}\right) \times$ $H_{0}^{1}\left(\Omega, \mathbb{R}_{\text {skew }}^{d \times d}\right)$ and $\mathcal{Z}=L^{2}\left(\Omega, \mathbb{R}_{\text {sym, dev }}^{d \times d}\right)$. It is proved in $[\mathrm{NC} 05]$ that there exists a constant $\alpha>0$ such that for all $(u, Q) \in \mathcal{U}$ and $\varepsilon_{p} \in \mathcal{Z}$ we have

$$
\mathcal{E}_{\mathrm{C}}\left((u, Q), \varepsilon_{p}\right) \geq \frac{\alpha}{2}\left(\|u\|_{H^{1}(\Omega)}^{2}+\|Q\|_{H^{1}(\Omega)}^{2}+\left\|\varepsilon_{p}\right\|_{L^{2}(\Omega)}^{2}\right)
$$

and therefore $\mathcal{E}_{\mathrm{C}}$ satisfies the assumption (2.18). Problem (2.2) formulated with $\mathcal{E}_{\mathrm{C}}$ from (5.3) and with a maximal monotone operator $\mathcal{G}$ defined as in (2.19) describes elasto-plastic material behavior which is coupled with Cosserat micropolar effects. Note that $D_{u} \mathcal{E}(u, z)$ in (2.2) has to be interpreted as $D_{(u, Q)} \mathcal{E}_{\mathrm{C}}\left(u, Q, \varepsilon_{p}\right)$. The existence of solutions was investigated in [NC05]. If $\mathbf{R} \mathbf{1}, \mathbf{R} 3$ and $\mathbf{R} 4$ are valid, then by Theorem 4.1 for every $\delta>0$ we have the global regularity $u \in L^{\infty}\left(S ; H^{\frac{3}{2}-\delta}(\Omega)\right)$ and $\varepsilon_{p} \in L^{\infty}\left(S ; H^{\frac{1}{2}-\delta}(\Omega)\right)$, while the existence proof already provides $Q \in W^{1,1}\left(S ; H^{2}(\Omega)\right)$, see [NC05]. It is also possible to choose $\mathcal{U}=H_{0}^{1}\left(\Omega, \mathbb{R}^{d}\right) \times H^{1}\left(\Omega ; \mathbb{R}_{\text {skew }}^{d \times d}\right)$, which means that Dirichlet conditions are prescribed for the displacements and Neumann conditions for the micro-rotation tensor $Q$.

\subsection{Discussion of the optimality of Theorem 4.1}

It is not clear whether the result presented in Theorem 4.1 is optimal or whether one should expect that $u \in L^{\infty}\left(S ; H^{2}(\Omega)\right)$ and $z \in L^{\infty}\left(S ; H^{1}(\Omega)\right)$ for smooth domains. The latter regularity would fit well to the local result provided in Theorem 3.1 and to regularity results for elliptic equations. In this section we show that the improved regularity can be achieved for some special cases. For $A \in C^{0,1}\left(\bar{\Omega} ; \operatorname{End}\left(\mathbb{R}^{m \times d} \times \mathbb{R}^{n}\right)\right)$ we use the notation $A=\left(\begin{array}{ll}A_{11} & A_{12} \\ A_{21} & A_{22}\end{array}\right)$ with coefficient matrices $A_{11} \in C^{0,1}\left(\bar{\Omega} ; \operatorname{End}\left(\mathbb{R}^{m \times d}\right)\right), A_{12}=A_{21}^{*} \in C^{0,1}\left(\bar{\Omega} ; \operatorname{Lin}\left(\mathbb{R}^{m \times d}, \mathbb{R}^{n}\right)\right)$ and $A_{22} \in C^{0,1}\left(\bar{\Omega} ; \operatorname{End}\left(\mathbb{R}^{n}\right)\right)$.

\subsubsection{The decoupled case}

Assume that $A_{12}=0$ and that $\mathcal{G}$ satisfies R3. In this case, the elliptic equation and the evolution equation in (4.2) are decoupled. The extended function $\ell_{2, e}$ occurring in the 
proof of Lemma 3.5 is now given by

$$
\ell_{2, e}(t, x)= \begin{cases}\ell_{2}(t) & x \in K_{r} \\ \ell_{2}(t, R x) & x \in C_{r} \backslash K_{r}\end{cases}
$$

and belongs to $W^{1,1}\left(S ; H^{1}\left(C_{r}\right)\right)$ instead of $L^{\infty}\left(S ; \mathcal{F}_{d}\left(C_{r}, C_{\rho}\right)\right)$. Thus part (b) of Theorem 3.1 is applicable and yields $z \in L^{\infty}\left(S ; H^{1}\left(K_{r}\right)\right)$. Under the assumptions of Theorem 4.1 it therefore holds in the decoupled case that $u \in W^{1,1}\left(S ; H^{2}(\Omega)\right)$ and $z \in L^{\infty}\left(S ; H^{1}(\Omega)\right)$.

\subsubsection{The one dimensional case}

Let $d=1, m, n \in \mathbb{N}$ and $\Omega=(0,1)$. Furthermore, let the pair $(u, z) \in W^{1,1}\left(S ; H^{1}(\Omega) \times\right.$ $L^{2}(\Omega)$ ) be a solution of (4.2). Applying the reflection procedure from Section 3.3 leads to extended functions having the regularity $\left(u_{e}, z_{e}\right) \in W^{1,1}\left(S ; H^{1}\left(C_{1}\right) \times L^{2}\left(C_{1}\right)\right), f_{e} \in$ $W^{1,1}\left(S ; L^{2}\left(C_{1}\right)\right), \theta_{e}, H_{e}, \ell_{2, e} \in W^{1,1}\left(S ; H^{1}\left(C_{1}\right)\right)$ with $C_{1}=(-1,1)$. Hence, Theorem 3.1(b) gives

Theorem 5.1. Let $d=1$ and assume that $\mathbf{R} 1-\mathbf{R} 4$ are satisfied. Then the solutions $u$ and $z$ have the regularity $u \in L^{\infty}\left(S ; H^{2}(\Omega)\right), z \in L^{\infty}\left(S ; H^{1}(\Omega)\right)$.

\subsubsection{Improved regularity if $u$ is scalar}

If the function $u$ is scalar, i.e. $m=1$, improved regularity results can be obtained provided that certain coupling conditions between the coefficient matrix $A$ and the function $g$ are satisfied. For the proof of the result we apply again a reflection argument. In contrast to the approach presented in Section 3 the model is not reflected perpendicular to the boundary but in a direction which is locally given by $A_{11}(x) \nu(x)$ for $x \in \partial \Omega$. Here, $\nu: \partial \Omega \rightarrow \mathbb{R}^{d}$ denotes the interior unit normal vector. In particular we assume

$\mathbf{R 1}{ }^{\prime} \Omega \subset \mathbb{R}^{d}$ is a bounded domain with $C^{2,1}$-smooth boundary and $\partial \Omega=\Gamma_{\mathrm{D}}$.

R2' The coefficient matrix $A$ belongs to $C^{1,1}\left(\bar{\Omega} ; \operatorname{End}\left(\mathbb{R}^{d} \times \mathbb{R}^{n}\right)\right)$, is self adjoint and satisfies $\mathcal{E}(v, z) \geq \frac{\alpha}{2}\left(\|v\|_{H^{1}(\Omega)}^{2}+\|z\|_{L^{2}(\Omega)}^{2}\right)$ for every $v \in H_{0}^{1}(\Omega)$ and $z \in L^{2}(\Omega)$.

R3' $g: \mathbb{R}^{n} \rightarrow \mathcal{P}\left(\mathbb{R}^{n}\right)$ satisfies $\mathbf{R 3}$.

R4' $z_{0} \in H^{1}\left(\Omega, \mathbb{R}^{n}\right), f \in W^{1,1}\left(S ; L^{2}(\Omega)\right)$ and $u_{0} \in W^{1,1}\left(S ; H^{2}(\Omega)\right)$.

In order to formulate the compatibility conditions, we define for $x \in \partial \Omega$ and $A_{11}(x) \in \mathbb{R}^{d \times d}$

$$
R_{\nu}(x)=\mathbb{I}-\frac{2}{\left\langle A_{11}(x) \nu(x), \nu(x)\right\rangle} A_{11}(x) \nu(x) \otimes \nu(x)
$$


with the interior normal vector $\nu: \partial \Omega \rightarrow \mathbb{R}^{d}$. The matrix $R_{\nu}$ locally determines the reflection at $\partial \Omega$. Observe that for all $x \in \partial \Omega$ we have

$$
\left(R_{\nu}(x)\right)^{2}=\mathbb{I} \quad \text { and } \quad R_{\nu}(x) A_{11}(x) R_{\nu}(x)^{\top}=A_{11}(x)
$$

R5' For every $x_{0} \in \partial \Omega$ there exists a neighborhood $W \subset \mathbb{R}^{d}$ and a mapping $P \in C^{0,1}(\partial \Omega \cap$ $W ; \operatorname{End}\left(\mathbb{R}^{n}\right)$ ) such that the inverse matrix $(P(x))^{-1}$ exists for every $x \in \partial \Omega \cap W$ and such that the following conditions hold for every $x \in \partial \Omega \cap W$ :

(a) $R_{\nu}(x) A_{12}(x) P(x)=A_{12}(x)$,

(b) $P(x)^{\top} A_{22}(x) P(x)=A_{22}(x)$,

(c) $-P(x)^{-1} g\left(-P(x)^{-\top} \eta\right)=g(\eta)$ for all $\eta \in \mathbb{R}^{n}$,

(d) Compatibility for the initial datum: $\left(\mathbb{I}+P^{-1}\right) z_{0}=0$ on $\partial \Omega$.

We consider the problem to find $(u, z) \in W^{1,1}\left(S ; H^{1}(\Omega)\right) \times W^{1,1}\left(S ; L^{2}(\Omega)\right)$ which satisfy for a.e. $t \in S$ and every $v \in H_{0}^{1}(\Omega)$ the relations

$$
\begin{array}{r}
\int_{\Omega}\left\langle A\left(\begin{array}{c}
\nabla u(t) \\
z(t)
\end{array}\right),\left(\begin{array}{c}
\nabla v \\
0
\end{array}\right)\right\rangle \mathrm{d} x=\int_{V} f(t) v \mathrm{~d} x, \\
\partial_{t} z(t) \in g\left(-\mathbb{P}_{n}\left[A\left(\begin{array}{c}
\nabla u(t) \\
z(t)
\end{array}\right)\right]\right) \\
z(0)=z_{0},\left.\quad u(t)\right|_{\partial \Omega}=\left.u_{0}(t)\right|_{\partial \Omega} .
\end{array}
$$

Theorem 5.2. Let R1'-R5' be satisfied and assume that the pair (u,z) solves (5.7). Then $u \in L^{\infty}\left(S ; H^{2}(\Omega)\right), z \in L^{\infty}\left(S ; H^{1}(\Omega)\right)$ and $\left(\mathbb{I}+P^{-1}\right) z(t)=0$ on $\partial \Omega$ for all $t \in S$.

The proof is carried out in the next two lemmata, where we first construct a local diffeomorphism from $\Omega \cap W$ to $W \backslash \Omega$. This diffeomorphism is closely related with $R_{\nu}$. In the second step we localize and extend problem (5.7) from $\Omega \cap W$ to $W$ and show that the new problem satisfies the smoothness assumptions of part (b) of Theorem 3.1.

Lemma 5.3. For every $x_{0} \in \partial \Omega$ exists a neighborhood $V$ with $V_{+}=\Omega \cap V$ and $V_{-}=V \backslash \bar{\Omega}$ and a $C^{1,1}$-diffeomorphism $T: V \rightarrow V$ with the properties $T(x)=x$ for all $x \in \partial \Omega \cap V$, $T\left(V_{ \pm}\right)=V_{\mp}$ and $\nabla T(x)=R_{\nu}(x)$ for all $x \in \partial \Omega \cap V$.

Proof. We define the following mapping

$$
\widetilde{T}: \partial \Omega \times \mathbb{R} \rightarrow \mathbb{R}^{d} ; \quad\left(\widetilde{y}, y_{d}\right) \mapsto \widetilde{y}+y_{d} A_{11}(\widetilde{y}) \nu(\widetilde{y})
$$


Since $\partial \Omega$ is assumed to be $C^{2,1}$-smooth, the mapping $\widetilde{T}$ belongs to $C^{1,1}\left(\partial \Omega \times \mathbb{R}^{d}\right)$. For $y_{d}=0$ we have

$$
\nabla \widetilde{T}(\widetilde{y}, 0)=\mathbb{I}_{\mathrm{T}_{\widetilde{y}} \partial \Omega}+A_{11}(\widetilde{y}) \nu(\widetilde{y}) \otimes \nu(\widetilde{y})=\mathbb{I}_{\mathbb{R}^{d}}+\left(A_{11}(\widetilde{y})-\mathbb{I}_{\mathbb{R}^{d}}\right) \nu(\widetilde{y}) \otimes \nu(\widetilde{y}),
$$

where $\mathbb{I}_{\mathrm{T}_{\widetilde{u}} \partial \Omega}$ is the restriction of the identity to the tangent space of $\partial \Omega$ in $\widetilde{y}$. Moreover, $\left.\operatorname{det} \nabla \widetilde{T}(\widetilde{y}, 0)=\left\langle A_{11}(\widetilde{y}) \nu(\widetilde{y})\right), \nu(\widetilde{y})\right\rangle>0$ since $A_{11}$ is uniformly positive definite. Thus the inverse of $\nabla \widetilde{T}(\widetilde{y}, 0)$ exists in all points $(\widetilde{y}, 0) \in \partial \Omega \times \mathbb{R}$ and is given by

$$
(\nabla \widetilde{T}(\widetilde{y}, 0))^{-1}=\mathbb{I}_{\mathbb{R}^{d}}-\frac{1}{\left\langle A_{11} \nu, \nu\right\rangle}\left(A_{11}-\mathbb{I}_{\mathbb{R}^{d}}\right) \nu \otimes \nu .
$$

Let now $x_{0} \in \partial \Omega$ be arbitrary. By the Implicit Function Theorem there exists a neighborhood $V \subset \mathbb{R}^{d}$ of $x_{0}$ and a neighborhood $\widetilde{V} \subset \partial \Omega \times \mathbb{R}$ of $\left(x_{0}, 0\right)$ such that $\widetilde{T}: \widetilde{V} \rightarrow V$ is a $C^{1,1}$-diffeomorphism with $\widetilde{T}\left(\widetilde{V}_{ \pm}\right)=V_{ \pm}$. Here, $\widetilde{V}_{ \pm}=\left\{\left(\widetilde{y}, y_{d}\right) \in \widetilde{V} ; y_{d} \geq 0\right\}, V_{+}=\Omega \cap V$, $V_{-}=V \backslash \bar{\Omega}$. Let the reflection at $\partial \Omega$ be given by $R: \partial \Omega \times \mathbb{R} \rightarrow \partial \Omega \times \mathbb{R}, R\left(\widetilde{y}, y_{d}\right)=\left(\widetilde{y},-y_{d}\right)$ with $\nabla R(\widetilde{y}, 0)=\mathbb{I}_{\mathbb{R}^{d}}-2 \nu \times \nu$. The mapping $T$ we are looking for is defined through

$$
T: V \rightarrow V, \quad T(x)=\widetilde{T}\left(R\left(\widetilde{T}^{-1}(x)\right)\right)
$$

By construction, $T$ is a $C^{1,1}$-diffeomorphism with $T\left(V_{ \pm}\right)=V_{\mp}$. Moreover, straightforward calculations show that for every $x \in \partial \Omega$ we have

$$
\nabla T(x)=\nabla \widetilde{T}(x, 0) \nabla R(x, 0)(\nabla \widetilde{T}(x, 0))^{-1}=R_{\nu}(x) .
$$

This finishes the proof of Lemma 5.3.

From now on we assume that $u_{0}=0$ and $u(t) \in H_{0}^{1}(\Omega)$ and that the set $V$ from Lemma 5.3 is contained in the set $W$ from R5'.

The following extended functions will be considered in the sequel: Choose $x_{0} \in \partial \Omega$ and let $T: V \rightarrow V$ be the corresponding diffeomorphism from Lemma 5.3. Choose $\varphi \in C_{0}^{\infty}(V)$ with $\left.\varphi\right|_{B_{\delta}\left(x_{0}\right)}=1$ for some $\delta>0$. The matrix valued function $P$ introduced in condition $\mathbf{R 5}^{\prime}$ is extended to $V$ in the following way: Let $\widetilde{T}$ be the diffeomorphism defined in the proof of Lemma 5.3. For $x \in V$ we have $\widetilde{T}^{-1}(x)=\left(\widetilde{y}, y_{d}\right) \in \partial \Omega \times \mathbb{R}$. By $\widetilde{T}_{\partial \Omega}^{-1}$ we denote the projection onto the point $\widetilde{y}$, i.e. $\widetilde{T}_{\partial \Omega}^{-1}(x)=\widetilde{y} \in \partial \Omega$. The extension of $P$ is now defined as

$$
P_{e}(x)=P\left(\widetilde{T}_{\partial \Omega}^{-1}(x)\right), \quad x \in V .
$$


By construction, $P_{e} \in C^{0,1}\left(\bar{V}, \operatorname{End}\left(\mathbb{R}^{n}\right)\right)$. Observe that the inverse matrix $\left(P_{e}(x)\right)^{-1}$ exists for every $x \in \bar{V}$ and that $\left(P_{e}(\cdot)\right)^{-1}$ belongs to $C^{0,1}\left(\bar{V}, \operatorname{End}\left(\mathbb{R}^{n}\right)\right)$. We define

$$
\begin{aligned}
& u_{e}(t, x)= \begin{cases}(\varphi u)(t, x) & (t, x) \in S \times V_{+} \\
-(\varphi u)\left(t, T^{-1}(x)\right) & (t, x) \in S \times V_{-}\end{cases} \\
& z_{e}(t, x)= \begin{cases}z(t, x) & (t, x) \in S \times V_{+} \\
-\left(P_{e}^{-1} z\right)\left(t, T^{-1}(x)\right) & (t, x) \in S \times V_{-}\end{cases}
\end{aligned}
$$

Obviously, $\left(u_{e}, z_{e}\right) \in W^{1,1}\left(S ; H_{0}^{1}(V) \times L^{2}(V)\right)$. The coefficient function $A$ is extended as follows

$$
\begin{aligned}
& A_{11, e}=\left\{\begin{array}{ll}
A_{11} & \text { on } V_{+} \\
\left(\nabla T A_{11} \nabla T^{\top}\right) \circ T^{-1} & \text { on } V_{-}
\end{array}, \quad A_{22, e}=\left\{\begin{array}{ll}
A_{22} & \text { on } V_{+} \\
\left(P_{e}^{\top} A_{22} P_{e}\right) \circ T^{-1} & \text { on } V_{-}
\end{array},\right.\right. \\
& A_{12, e}=\left\{\begin{array}{ll}
A_{12} & \text { on } V_{+} \\
\left(\nabla T A_{12} P_{e}\right) \circ T^{-1} & \text { on } V_{-}
\end{array}, \quad A_{21, e}=A_{12, e}^{\top} .\right.
\end{aligned}
$$

Due to the compatibility condition R5', the coefficient matrix $A_{e}=\left(\begin{array}{cc}A_{11, e} & A_{12, e} \\ A_{21, e} & A_{22, e}\end{array}\right)$ belongs to $C^{0,1}\left(\bar{V}, \operatorname{End}\left(\mathbb{R}^{d} \times \mathbb{R}^{n}\right)\right)$. Moreover, the data is extended as follows

$$
\begin{aligned}
& f_{e}=\left\{\begin{array}{ll}
f & \text { on } V_{+} \\
\left(-f+|\operatorname{det} \nabla T|^{-1}\left(A_{11} \nabla u+A_{12} z\right) \cdot \nabla|\operatorname{det} \nabla T|\right) \circ T^{-1} & \text { on } V_{-}
\end{array},\right. \\
& H_{e}=\left\{\begin{array}{ll}
A_{11} \nabla((\varphi-1) u) & \text { on } V_{+} \\
-\left(\nabla T A_{11} \nabla((\varphi-1) u)\right) \circ T^{-1} & \text { on } V_{-}
\end{array},\right. \\
& \ell_{2, e}= \begin{cases}A_{21} \nabla((\varphi-1) u) & \text { on } V_{+} \\
-\left(P_{e}^{\top} A_{21} \nabla((\varphi-1) u)\right) \circ T^{-1} & \text { on } V_{-}\end{cases} \\
& z_{0, e}= \begin{cases}z_{0} & \text { on } V_{+} . \\
-\left(P_{e}^{-1} z_{0}\right) \circ T^{-1} & \text { on } V_{-}\end{cases}
\end{aligned}
$$

Thanks to R5', the extended functions have the regularity $f_{e} \in W^{1,1}\left(S ; L^{2}(V)\right), H_{e}, \ell_{2, e} \in$ $W^{1,1}\left(S ; L^{2}(V)\right) \cap W^{1,1}\left(S ; H^{1}\left(B_{\delta}\left(x_{0}\right)\right)\right)$ and $z_{0, e} \in H^{1}(V)$. Finally, for $\eta \in \mathbb{R}^{n}$ we define

$$
g_{e}(x, \eta)= \begin{cases}g(\eta) & \text { on } V_{+} \\ -P_{e}^{-1} \circ T^{-1} g\left(-P_{e}^{-\top} \circ T^{-1} \eta\right) & \text { on } V_{-} .\end{cases}
$$

Due to condition R5', we have in fact the identity $g_{e}(x, \eta)=g(\eta)$ for all $x \in V$ and $\eta \in \mathbb{R}^{n}$. 
Lemma 5.4. Assume that R1'-R5' of this section are satisfied. For a.e. $t \in S$ and every $v \in H_{0}^{1}(V)$ the above defined extended functions $\left(u_{e}, z_{e}\right) \in W^{1,1}\left(S ; H_{0}^{1}(V)\right) \times W^{1,1}\left(S ; L^{2}(V)\right)$ satisfy

$$
\begin{aligned}
\int_{V}\left\langle A_{e}\left(\begin{array}{c}
\nabla u_{e}(t) \\
z_{e}(t)
\end{array}\right),\left(\begin{array}{c}
\nabla v \\
0
\end{array}\right)\right\rangle \mathrm{d} x & =\int_{V} f_{e}(t) v+H_{e}(t) \cdot \nabla v \mathrm{~d} x, \\
\partial_{t} z_{e}(t) & \in g_{e}\left(-\mathbb{P}_{n}\left[A_{e}\left(\begin{array}{c}
\nabla u_{e}(t) \\
z_{e}(t)
\end{array}\right)\right]+\ell_{2, e}(t)\right) \\
z_{e}(0) & =z_{0, e} .
\end{aligned}
$$

Moreover, the coefficients and the data $z_{0, e}, f_{e}, H_{e}$ and $\ell_{2, e}$ have the smoothness described in conditions $\mathbf{A} 1$ and $\mathbf{A} 3$ of Section 3.1. Thus, $u \in L^{\infty}\left(S ; H^{2}\left(B_{\delta}\left(x_{0}\right) \cap \Omega\right)\right)$ and $z \in$ $L^{\infty}\left(S ; H^{1}\left(B_{\delta}\left(x_{0}\right) \cap \Omega\right)\right)$.

Proof. The last assertion of Lemma 5.4 is an immediate consequence of Theorem 3.1 applied to $u_{e}$ and $z_{e}$ and (5.9). We recall that $g_{e}(x, \eta)=g(\eta)$ for all $x \in V$ and $\eta \in \mathbb{R}^{n}$. Since $z_{e} \in L^{\infty}\left(S ; H^{1}\left(B_{\delta}\left(x_{0}\right)\right)\right.$, the traces of $z_{e}$ from $V_{+}$and from $V_{-}$on $\partial \Omega$ coincide, which entails $\left(\mathbb{I}+P^{-1}\right) z(t)=0$ on $\partial \Omega$ for all $t$.

Relation (5.9) can be derived as follows: straightforward calculations show that for $v \in H_{0}^{1}(V)$ it holds

$$
\begin{aligned}
\int_{V}\left\langle A_{e}\left(\begin{array}{c}
\nabla u_{e}(t) \\
z_{e}(t)
\end{array}\right),\left(\begin{array}{c}
\nabla v \\
0
\end{array}\right)\right\rangle \mathrm{d} x= & \int_{V_{+}}\left(A_{11} \nabla u_{e}+A_{12} z\right) \cdot \nabla(v-|\operatorname{det} \nabla T| v \circ T) \mathrm{d} y \\
& +\int_{V_{+}}\left(\left(A_{11} \nabla u_{e}+A_{12} z\right) \cdot \nabla|\operatorname{det} \nabla T|\right) v \circ T \mathrm{~d} y .
\end{aligned}
$$

Since $|\operatorname{det} \nabla T(y)|=\left|\operatorname{det} R_{\nu}(y)\right|=1$ for $y \in \partial \Omega$, it follows that $v-|\operatorname{det} \nabla T| v \circ T \in H_{0}^{1}\left(V_{+}\right)$. Thus, on the basis of (5.7), we arrive at the following relation

$$
\begin{aligned}
\int_{V}\left\langle A_{e}\left(\begin{array}{c}
\nabla u_{e}(t) \\
z_{e}(t)
\end{array}\right),\left(\begin{array}{c}
\nabla v \\
0
\end{array}\right)\right\rangle \mathrm{d} x= & \int_{V_{+}} f(v-|\operatorname{det} \nabla T| v \circ T)+H_{e} \cdot \nabla(v-|\operatorname{det} \nabla T| v \circ T) \mathrm{d} y \\
& +\int_{V_{-}}\left(|\operatorname{det} \nabla T|^{-1}\left(A_{11} \nabla u_{e}+A_{12} z\right) \cdot \nabla|\operatorname{det} \nabla T|\right) \circ T^{-1} v \mathrm{~d} x
\end{aligned}
$$

with $H_{e}$ from above. After a transformation of coordinates we obtain the first relation in Lemma 5.4. The second relation is an immediate consequence of the definitions of the extended functions in combination with relation (5.7).

A concrete example, where condition R5' is satisfied, is the following with $n=d$ :

Let $A=\left(\begin{array}{cc}A_{11} & -A_{11} \\ -A_{11} & A_{11}+A_{22}\end{array}\right)$ with symmetric, positive definite and constant matrices $A_{11}$, $A_{22} \in \mathbb{R}^{d \times d}$. The corresponding stored energy reads

$$
\mathcal{E}(u, z)=\frac{1}{2} \int_{\Omega} A_{11}(\nabla u-z) \cdot(\nabla u-z)+A_{22} z \cdot z \mathrm{~d} x .
$$


Moreover, $g=\partial \chi_{\mathcal{K}}$ for some convex and closed set $\mathcal{K} \subset \mathbb{R}^{d}$. Let $\Omega \subset \mathbb{R}^{d}$ satisfy $\mathbf{R} 1$ ' and let $R_{\nu}:=\mathbb{I}-\frac{2}{\left\langle A_{11} \nu, \nu\right\rangle} A_{11} \nu \otimes \nu$ for $\nu \in \mathbb{R}^{d} \backslash\{0\}$. Observe that $R_{\nu}^{-1}=R_{\nu}$. With $P_{\nu}=A_{11}^{-1} R_{\nu}^{-1} A_{11}=R_{\nu}^{\top}$, condition $\mathbf{R} 5$ ' reads as follows

(a) $R_{\nu} A_{22} R_{\nu}^{\top}=A_{22}$ for all $\nu \in \mathbb{R}^{d} \backslash\{0\}$,

(b) $-R_{\nu}^{\top} g\left(-R_{\nu} \eta\right)=g(\eta)$ for all $\eta \in \mathbb{R}^{d}$ and $\nu \in \mathbb{R}^{d} \backslash\{0\}$.

Proposition 5.5. The compatibility condition R5' is satisfied if and only if $\mathcal{K}=-R_{\nu} \mathcal{K}$ for every $\nu \in \mathbb{R}^{d} \backslash\{0\}$ and if there exists $\alpha>0$ such that $A_{22}=\alpha A_{11}$.

If $\mathcal{K}=\left\{\eta \in \mathbb{R}^{d} ;\langle B \eta, \eta\rangle \leq 1\right\}$ for some symmetric and positive definite $B \in \mathbb{R}^{d \times d}$, then the condition on $\mathcal{K}$ is satisfied if and only if there exists a constant $\beta>0$ such that $B=\beta A_{11}^{-1}$.

Proof. The Proposition follows from Lemma A.1 and Lemma A.2 in the appendix.

This scalar example shows that if the anisotropy of "Hooke's law" given by $A_{11}$ is strongly correlated with the anisotropy in the hardening coefficients $A_{22}$ and the convex set $\mathcal{K}$, then the displacements $u(t)$ have full $H^{2}$ regularity up to the boundary of $\Omega$. The crucial point in the scalar case is the existence of the local diffeomorphism $T$ from $\Omega$ to some larger domain having the property (5.6) for $R_{\nu}=\nabla T$. It is not clear, whether a similar construction is possible for true elasto-plasticity, where $m=d$, or for the general vectorial case with $m>1$.

Example 5.6. As a byproduct of the proof of Theorem 5.2 we obtain the following regularity result, which might be of interest for convergence studies of numerical schemes. Let $\Omega \subset \mathbb{R}^{d}$ be a rectangular cuboid, $m=1, n=d, \mathcal{E}(u, z)=\frac{1}{2} \int_{\Omega}|\nabla u-z|^{2}+\alpha|z|^{2} \mathrm{~d} x$ for some $\alpha>0$ and $g=\partial \chi_{K}$ with $K=\bar{B}_{\rho}(0) \subset \mathbb{R}^{d}$. Assume that $\mathbf{R} 4$ ' is satisfied. If in addition there exists $\xi: \partial \Omega \rightarrow \mathbb{R}$ such that the initial datum satisfies $z_{0}(x)=\xi(x) \nu(x)$, where $\nu: \partial \Omega \rightarrow \mathbb{R}^{d}$ is the interior normal vector, then $u \in L^{\infty}\left(S ; H^{2}(\Omega)\right)$ and $z \in L^{\infty}\left(S ; H^{1}(\Omega)\right)$.

Proof. Like in the proof of Theorem 5.2 we extend the problem by reflection across all faces to a larger cuboid which completely contains $\Omega$. Due to the compatibility assumptions on the coefficients and on the initial datum the extended data and coefficients are smooth and part (b) of Theorem 3.1 is applicable.

\subsubsection{Example: $\partial_{t} z \notin L^{\infty}\left(S ; H^{1}(\Omega)\right)$}

In spite of smooth data the rate $\partial_{t} z$ does not belong to $L^{\infty}\left(S ; H^{1}(\Omega)\right)$, in general. The following example is inspired by Seregin's paper [Ser99]. 
Let $0<R_{1}<R_{2}$. We set $\Omega=B_{R_{2}}(0) \backslash B_{R_{1}}(0)$ and choose the following energy for $u, z: \Omega \rightarrow \mathbb{R}:$

$$
\mathcal{E}(u, z)=\frac{1}{2} \int_{\Omega}\left|\nabla u-\frac{x}{|x|} z\right|^{2}+z^{2} \mathrm{~d} x .
$$

Moreover, $g(\eta):=\partial \chi_{[-1,1]}(\eta)$ for $\eta \in \mathbb{R}$. We assume that $\left.u(t)\right|_{\partial B_{R_{1}}}=0,\left.u(t)\right|_{\partial B_{R_{2}}}=t$, $z_{0}=0$ and that the remaining data $\left(f, H, \ell_{2}\right)$ vanish. It is easily checked that the assumptions of Theorem 5.2 are satisfied and hence the problem has a unique solution with the regularity $\nabla u, z \in W^{1,1}\left(S ; L^{2}(\Omega)\right) \cap L^{\infty}\left(S ; H^{1}(\Omega)\right)$. Due to the rotational symmetry of the problem the solution does not depend on the angle and can explicitly be calculated. Introducing polar-coordinates, the solution $u, z: S \times\left(R_{1}, R_{2}\right) \rightarrow \mathbb{R}$ has to satisfy

$$
\begin{gathered}
\partial_{r}^{2} u+r^{-1} \partial_{r} u-\partial_{r} z-r^{-1} z=0 \quad \text { in } S \times\left(R_{1}, R_{2}\right), \\
\partial_{t} z \in \partial \chi_{[-1,1]}\left(\partial_{r} u-2 z\right) \quad \text { in } S \times\left(R_{1}, R_{2}\right), \\
z(0, \cdot)=0, \quad u\left(t, R_{1}\right)=0, \quad u\left(t, R_{2}\right)=t .
\end{gathered}
$$

For $t \leq t_{1}:=R_{1} \ln \left(R_{2} / R_{1}\right)$ it follows that $u(t, r)=\frac{t \ln \left(r / R_{1}\right)}{\ln \left(R_{2} / R_{1}\right)}, z(t, r)=0$. In this regime, no plastic strains are present. For $t>t_{1}$ the plastic variable $z$ starts to grow and there exists $r_{*}(t)$ such that $z(t, r)>0$ for $r<r_{*}$ and $z(r, t)=0$ for $r>r_{*}$, i.e. $r_{*}(t)$ separates the plastic region from the elastic region. The dependence of $r_{*}$ of $t$ is given implicitly through the relation

$$
t\left(r_{*}\right)=R_{1}-r_{*}+r_{*} \ln \frac{R_{2} r_{*}}{R_{1}^{2}} .
$$

Simple calculations show that $t\left(r_{*}\right)$ is strictly increasing, and hence $r_{*}(t) \geq R_{1}$ is strictly growing, as well. Moreover, for $t \geq t_{1}$ we have

$$
u(t, r)=\left\{\begin{array}{ll}
b(t)-r+2 r_{*}(t) \ln r & \text { if } r \leq r_{*}(t) \\
c(t)+r_{*}(t) \ln r & \text { else }
\end{array}, z(t, r)=\left\{\begin{array}{ll}
-1+r_{*}(t) r^{-1} & \text { if } r \leq r_{*}(t), \\
0 & \text { else }
\end{array},\right.\right.
$$

with functions $b(t)=R_{1}-2 r_{*}(t) \ln R_{1}$ and $c(t)=t-r_{*}(t) \ln R_{2}$. Since $r_{*}^{\prime}(t)>0$ for $t \geq t_{1}$ it follows that $\partial_{t} z(t, \cdot) \notin H^{1}\left(R_{1}, R_{2}\right)$ for $t>t_{1}$. This example shows that in general one can at most expect $\partial_{t} z \in L^{p}\left(S ; H^{\frac{1}{2}-\delta}(\Omega)\right)$ for some $p \in[1, \infty]$ and $\delta>0$.

\section{Convergence rate for space-time-discretization}

In this section we demonstrate how the above proved regularity results enter into the derivation of convergence rates for a space-time-discretization of elasto-plastic models. 
For shortness we restrict ourselves to the case with Dirichlet boundary conditions, hence $\mathcal{U}=H_{0}^{1}(\Omega)$, and assume that the coefficient tensor $A$ is constant. Moreover, we consider the rate-independent case, where the maximal monotone function $\mathcal{G}$ coincides with the subdifferential of a characteristic function. Thus, classical elasto-plasticity with linear kinematic hardening is included in this section.

Let $\mathcal{T}_{h}$ be a regular triangulation of $\Omega$. For approximating the generalized displacements we use functions, which are continuous and piecewise affine with respect to $\mathcal{T}_{h}$, while for the interior variables we use piecewise constant functions:

$$
\begin{aligned}
& \mathcal{U}_{h}=\left\{u \in H_{0}^{1}(\Omega) ;\left.u\right|_{\tau} \in \mathbb{P}_{1}(\tau) \forall \tau \in \mathcal{T}_{h}\right\}, \\
& \mathcal{Z}_{h}=\left\{z \in L^{2}(\Omega) ;\left.z\right|_{\tau} \in \mathbb{P}_{0}(\tau) \forall \tau \in \mathcal{T}_{h}\right\} .
\end{aligned}
$$

Here, $\mathbb{P}_{k}(\tau)$ denote the polynomials of order $k$ on $\tau$. Let the energy $\mathcal{E}$ be given according to $\mathbf{R} 2$ in Section 4.

Let furthermore $K \subset \mathbb{R}^{n}$ be convex, closed and with $0 \in K, \mathcal{K}=\left\{z \in L^{2}\left(\Omega, \mathbb{R}^{n}\right) ; z(x) \in\right.$ $K$ a.e. in $\Omega\}$ and $\mathcal{K}_{h}=\mathcal{K} \cap \mathcal{Z}_{h}$. Let furthermore $\mathcal{G}=\partial_{\mathcal{Z}} \chi_{\mathcal{K}}$ be the subdifferential in $\mathcal{Z}$ of the characteristic function associated with $\mathcal{K}$ and $\mathcal{G}_{h}=\partial_{\mathcal{Z}_{h}} \chi_{\mathcal{K}_{h}}$ the subdifferential in $\mathcal{Z}_{h}$ of the characteristic function associated with $\mathcal{K}_{h}$. Observe that $\mathcal{G}, \mathcal{G}_{h}$ and the space $\mathcal{Z}_{h}$ satisfy the compatibility assumption (2.11). Indeed, for $\eta_{h} \in \mathcal{Z}_{h}$ we have the identity $\mathcal{G}_{h}\left(\eta_{h}\right)=\left\{\theta_{h} \in \mathcal{Z}_{h} ; \theta_{h}(x) \in \partial \chi_{K}\left(\eta_{h}(x)\right)\right.$ a.e. in $\left.\Omega\right\}=\mathcal{G}\left(\eta_{h}\right) \cap \mathcal{Z}_{h}$.

The problem to be approximated reads: Given compatible data $\left(z_{0}, f\right) \in \mathcal{Z} \times W^{1, \infty}\left(S ; H^{-1}(\Omega)\right)$ find $(u, z) \in W^{1, \infty}(S ; \mathcal{U} \times \mathcal{Z})$ with $z(0)=z_{0}$ such that for a.e. $t \in S$ and all $v \in \mathcal{U}$ it holds

$$
\begin{aligned}
\int_{\Omega}\left\langle A\left(\begin{array}{c}
\nabla u(t) \\
z(t)
\end{array}\right),\left(\begin{array}{c}
\nabla v \\
0
\end{array}\right)\right\rangle \mathrm{d} x & =\langle f(t), v\rangle, \\
\partial_{t} z(t) & \in \mathcal{G}\left(-D_{z} \mathcal{E}(u(t), z(t))\right) .
\end{aligned}
$$

The fully discretized problem is defined as: Given a partition $\Pi^{\tau}$ of the time-interval $[0, T]$ and a triangulation $\mathcal{T}_{h}$, find $u_{h, \tau}^{k} \in \mathcal{U}_{h}$ and $z_{h, \tau}^{k} \in \mathcal{Z}_{h}$ which for $1 \leq k \leq N^{\tau}$ and every $v \in \mathcal{U}_{h}$ satisfy

$$
\begin{aligned}
\int_{\Omega}\left\langle A\left(\begin{array}{c}
\nabla u_{h, \tau}^{k} \\
z_{h, \tau}^{k}
\end{array}\right),\left(\begin{array}{c}
\nabla v \\
0
\end{array}\right)\right\rangle \mathrm{d} x & =\left\langle f\left(t_{\tau}^{k}\right), v\right\rangle, \\
\frac{z_{h, \tau}^{k}-z_{h, \tau}^{k-1}}{t_{\tau}^{k}-t_{\tau}^{k-1}} & \in \mathcal{G}\left(-D_{z} \mathcal{E}\left(u_{h, \tau}^{k}, z_{h, \tau}^{k}\right)\right) \\
z_{h, \tau}^{0} & =z_{0, h} .
\end{aligned}
$$

Here, $z_{0, h}$ is a suitable projection of the initial datum $z_{0}$ on $\mathcal{Z}_{h}$. The fully discrete model corresponds to a conforming approximation of the convex set $\mathcal{K}$ and coincides with the 
discrete problem studied in [AC00, HR99]. Let $\rho=\chi_{K}^{*}: \mathbb{R}^{n} \rightarrow \mathbb{R}^{n}$ be the convex conjugate of $\chi_{K}$ and $\mathcal{R}(\eta)=\int_{\Omega} \rho(\eta(x)) \mathrm{d} x$ for $\eta \in \mathcal{Z}$. Then the discrete problem may equivalently be written as: Find $\left(u_{h, \tau}^{k}, z_{h, \tau}^{k}\right) \in \mathcal{U}_{h} \times \mathcal{Z}_{h}$ such that

$$
\left(u_{h, \tau}^{k}, z_{h, \tau}^{k}\right) \in \operatorname{Argmin}\left\{\mathcal{E}\left(v_{h}, \eta_{h}\right)-\left\langle f\left(t_{\tau}^{k}\right), v_{h}\right\rangle+\mathcal{R}\left(\eta_{h}-z_{h, \tau}^{k-1}\right) ; v_{h} \in \mathcal{U}_{h}, \eta_{h} \in \mathcal{Z}_{h}\right\} .
$$

This incremental minimization problem is the starting point for the convergence studies in [MRS08]. Using similar arguments as e.g. in [KN08], the problem (6.2) may also be written as a system of quasilinear elliptic equations for determining $u_{h, \tau}^{k}$ from $z_{h, \tau}^{k-1}$, which is combined with a simple update formula for $z_{h, \tau}^{k}$.

As in Section 2.2 we denote with $\hat{u}_{h, \tau}$ and $\hat{z}_{h, \tau}$ the continuous and piecewise affine interpolants corresponding to the time incremental solutions $\left\{\left(u_{h, \tau}^{k}, z_{h, \tau}^{k}\right) ; 0 \leq k \leq N^{\tau}\right\}$. For the error between the original solution $(u, z)$ and the approximation $\left(\hat{u}_{h, \tau}, \hat{z}_{k, \tau}\right)$ we obtain

Theorem 6.1. Under the above assumptions and if the coefficient tensor $A$ is constant satisfies $\mathbf{R 2}$ (Section 4), there exists a constant $c>0$ such that for all $h, \tau$ and all $t \in[0, T]$ it holds

$$
\begin{aligned}
c\left(\left\|u(t)-\hat{u}_{h, \tau}(t)\right\|_{H^{1}(\Omega)}+\left\|z(t)-\hat{z}_{h, \tau}(t)\right\|_{L^{2}(\Omega)}\right) & \\
\leq & \sqrt{\tau}+\tau\|f\|_{W^{1, \infty}\left(S ; \mathcal{U}^{*}\right)}+\inf _{v_{h} \in \mathcal{U}_{h}}\left\|u(t)-v_{h}\right\|_{\mathcal{U}} \\
& \quad+\left\|z_{0}-z_{0, h}\right\|_{L^{2}(\Omega)}+\sup _{s \in S} \inf _{v_{h} \in \mathcal{U}_{h}}\left\|u(s)-v_{h}\right\|_{H^{1}(\Omega)}^{\frac{1}{2}}
\end{aligned}
$$

If in particular, if $u \in L^{\infty}\left(S ; H^{1+s}(\Omega)\right)$ for some $s \in(0,1]$, and $\left\|z_{0}-z_{0, h}\right\|_{\mathcal{Z}} \leq$ ch $^{\frac{s}{2}}$, then we obtain the convergence rate

$$
\left\|u(t)-\hat{u}_{h, \tau}(t)\right\|_{H^{1}(\Omega)}+\left\|z(t)-\hat{z}_{h, \tau}(t)\right\|_{L^{2}(\Omega)} \leq c\left(\sqrt{\tau}+h^{\frac{s}{2}}\right) .
$$

Observe that $\left\|z-z_{h}\right\|_{W^{1,1}(S ; \mathcal{Z})}$ enters into the constant $c$ of estimate (6.3). Due to Theorem 2.4 this error is uniformly bounded with respect to $h$.

Proof. The Strang Lemma implies the following estimate for the difference of the original solution $u$ and the affine interpolant $\hat{u}_{h, \tau}$ : There exists a constant $c_{1}>0$ such that for all $h$ and $\tau$ and for all $t \in[0, T]$ it holds

$$
\left\|u(t)-\hat{u}_{h, \tau}(t)\right\|_{\mathcal{U}} \leq c_{1}\left(\inf _{v_{h} \in \mathcal{U}_{h}}\left\|u(t)-v_{h}\right\|_{\mathcal{U}}+\left\|\hat{z}_{h, \tau}(t)-z(t)\right\|_{\mathcal{Z}}+\left\|f(t)-\hat{f}_{\tau}(t)\right\|_{\mathcal{U}^{*}}\right)
$$

where $\hat{f}_{\tau} \in W^{1, \infty}\left(S ; \mathcal{U}^{*}\right)$ is the piecewise affine interpolant associated with $\left\{f\left(t_{\tau}^{k}\right) ; 1 \leq\right.$ $\left.k \leq N^{\tau}\right\}$. Hence it is sufficient to provide an estimate for $\left\|\hat{z}_{h, \tau}(t)-z(t)\right\|_{\mathcal{Z}}$. But this 
estimate relies on Corollary 2.5. In order to apply Corollary 2.5, we have to define the Schur complement operators $\mathcal{L}$ and $\mathcal{L}_{h}$ and prove that they satisfy condition (2.10) of Section 2.2.

The coefficient matrix $A \in \operatorname{End}\left(\mathbb{R}^{m \times d} \times \mathbb{R}^{n}\right)$ is split as follows: $A=\left(\begin{array}{ll}A_{11} & A_{12} \\ A_{21} & A_{22}\end{array}\right)$ with $A_{11} \in \operatorname{End}\left(\mathbb{R}^{m \times d}\right), A_{12}=A_{21}^{*} \in \operatorname{Lin}\left(\mathbb{R}^{n}, \mathbb{R}^{m \times d}\right)$ and $A_{22} \in \operatorname{End}\left(\mathbb{R}^{n}\right)$. The induced linear and bounded operators $\mathcal{A}_{i j}$ are given as follows:

$$
\begin{aligned}
\mathcal{A}_{11}: \mathcal{U} \rightarrow \mathcal{U}^{*}, & \left\langle\mathcal{A}_{11}(u), v\right\rangle=\int_{\Omega} A_{11} \nabla u: \nabla v \mathrm{~d} x \quad u, v \in \mathcal{U}, \\
\mathcal{A}_{11, h}: \mathcal{U}_{h} \rightarrow \mathcal{U}_{h}^{*}, & \left\langle\mathcal{A}_{11, h}\left(u_{h}\right), v_{h}\right\rangle=\int_{\Omega} A_{11} \nabla u_{h}: \nabla v_{h} \mathrm{~d} x \quad u_{h}, v_{h} \in \mathcal{U}_{h}, \\
\mathcal{A}_{12}=\mathcal{A}_{21}^{*}: \mathcal{Z} \rightarrow \mathcal{U}^{*}, & \left\langle\mathcal{A}_{12}(z), u\right\rangle=\int_{\Omega} A_{12} z: \nabla u \mathrm{~d} x \quad u \in \mathcal{U}, z \in \mathcal{Z}, \\
\mathcal{A}_{22}: \mathcal{Z} \rightarrow \mathcal{Z}, & \mathcal{A}_{22}(z)=A_{22} z \quad z \in \mathcal{Z} .
\end{aligned}
$$

Due to the positivity assumption $\mathbf{R} 2, \mathcal{A}_{11}$ and $\mathcal{A}_{11, h}$ are uniformly (with respect to $h$ ) positive definite on $\mathcal{U}$ and $\mathcal{U}_{h}$. Hence, the following Schur-complement operators are well defined and uniformly bounded with respect to $h$ :

$$
\begin{aligned}
\mathcal{L}: \mathcal{Z} \rightarrow \mathcal{Z}, & \mathcal{L}=\mathcal{A}_{22}-\mathcal{A}_{21} \mathcal{A}_{11}^{-1} \mathcal{A}_{12} \\
\mathcal{L}_{h}: \mathcal{Z} \rightarrow \mathcal{Z}, & \mathcal{L}_{h}=\mathcal{A}_{22}-\mathcal{A}_{21} \mathcal{A}_{11, h}^{-1} \mathcal{A}_{12}
\end{aligned}
$$

Moreover, $\mathcal{L}$ and $\mathcal{L}_{h}$ are self adjoint and uniformly positive definite on $\mathcal{Z}$. The uniform positive definiteness can be seen as follows: For all $z \in \mathcal{Z}$ we have

$$
\left\langle\mathcal{L}_{h} z, z\right\rangle=\left\langle\left(\begin{array}{cc}
\mathcal{A}_{11, h} & \mathcal{A}_{12} \\
\mathcal{A}_{21} & \mathcal{A}_{22}
\end{array}\right)\left(\underset{z}{-\mathcal{A}_{11, h}^{-1} \mathcal{A}_{12} z}\right),\left(\underset{z}{-\mathcal{A}_{11, h}^{-1} \mathcal{A}_{12} z}\right)\right\rangle \geq \alpha\left(\left\|\mathcal{A}_{11, h}^{-1} \mathcal{A}_{12} z\right\|_{\mathcal{U}}^{2}+\|z\|_{\mathcal{Z}}^{2}\right)
$$

with $\alpha>0$ from condition R2. Since $\mathcal{A}_{21}$ maps $\mathcal{U}_{h}$ to $\mathcal{Z}_{h}$ and since the coefficients $A$ are constant, the operator $\mathcal{L}_{h}$ maps $\mathcal{Z}_{h}$ onto $\mathcal{Z}_{h}$. Hence, assumption (2.10) is satisfied. Finally, we define $F \in W^{1, \infty}(S ; \mathcal{Z})$ and $F_{h} \in W^{1, \infty}\left(S ; \mathcal{Z}_{h}\right)$ through the relations $F(t)=$ $-\mathcal{A}_{21} \mathcal{A}_{11}^{-1} f(t)$ and $F_{h}(t)=-\mathcal{A}_{21} \mathcal{A}_{11, h}^{-1} f(t)$. With these definitions we are exactly in the situation described in Section 2.2 and problem (6.2) is equivalent to problem (2.15).

Hence, the error estimate between the original solution $z$ and the affine interpolation of the solutions of the fully discretized problem provided in Corollary 2.5 is valid. It remains to derive an estimate for the term $\left\|(\mathcal{L} z-F)-\left(\mathcal{L}_{h} z-F_{h}\right)\right\|_{L^{\infty}(S ; \mathcal{Z})}$. Observe that for all $t \in[0, T]$

$$
\left\|(\mathcal{L} z-F)(t)-\left(\mathcal{L}_{h} z-F_{h}\right)(t)\right\|_{\mathcal{Z}} \leq c\left\|u(t)-\tilde{u}_{h}(t)\right\|_{\mathcal{U}}
$$


where $u$ is the solution of the original problem (6.1) and $\tilde{u}_{h} \in L^{\infty}\left(S, \mathcal{U}_{h}\right)$ satisfies

$$
\int_{\Omega} A_{11} \nabla \tilde{u}_{h}(t): \nabla v_{h} \mathrm{~d} x=\left\langle f(t), v_{h}\right\rangle-\int_{\Omega} A_{12} z(t): \nabla v_{h} \mathrm{~d} x
$$

for all $v_{h} \in \mathcal{U}_{h}$ and $t \in[0, T]$. Hence, Céa's Lemma implies that there is a constant $c>0$ such that for all $t$ it holds

$$
\left\|u(t)-\tilde{u}_{h}(t)\right\|_{\mathcal{U}} \leq \inf _{v_{h} \in \mathcal{U}_{h}}\|u(t)-v\|_{\mathcal{U}}
$$

Collecting all estimates finally finishes the proof of Theorem 6.1.

\section{A Proof of Proposition 5.5}

Lemma A.1. Let $A, B \in \operatorname{Lin}\left(\mathbb{R}^{d}, \mathbb{R}^{d}\right)$ be symmetric with $\operatorname{det} A \neq 0$ and assume that for all $\nu \in \mathbb{R}^{d} \backslash\{0\}$ we have $R_{\nu} B R_{\nu}^{\top}=B$ with $R_{\nu}=\mathbb{I}-\frac{2}{\langle A \nu, \nu\rangle} A \nu \otimes \nu$. Then there exists $\alpha \in \mathbb{R}$ such that $B=\alpha A$.

Proof. Let $\left\{e_{1}, \ldots, e_{d}\right\}$ be an orthonormal system of eigenvectors of $A$, i.e. $A e_{i}=\lambda_{i} e_{i}$ for some $\lambda_{i} \in \mathbb{R} \backslash\{0\}$ and $\left\langle e_{i}, e_{j}\right\rangle=\delta_{i j}$. Then the set $\left\{e_{i} \otimes e_{j} ; i, j \in\{1, \ldots, d\}\right\}$ is a basis of $\operatorname{Lin}\left(\mathbb{R}^{d}, \mathbb{R}^{d}\right)$ which is orthonormal with respect to the inner product defined by $S: T=\operatorname{tr}\left(T^{\top} S\right)$. This means that $\left(e_{i} \otimes e_{j}\right):\left(e_{k} \otimes e_{l}\right)=\delta_{i k} \delta_{j l}$ and $A:\left(e_{i} \otimes e_{j}\right)=\lambda_{i} \delta_{i j}$. Thus the identity $R_{\nu} B R_{\nu}^{\top}=B$ is valid for all $\nu \in \mathbb{R}^{d} \backslash\{0\}$ if and only if

$$
\left(R_{\nu} B R_{\nu}^{\top}\right):\left(e_{i} \otimes e_{j}\right)=B:\left(e_{i} \otimes e_{j}\right)
$$

for all $\nu \in \mathbb{R}^{d} \backslash\{0\}$ and all $i, j \in\{1, \ldots, d\}$. Observe that (A.1) is equivalent to

$$
2 \lambda_{i} \lambda_{j}\left\langle\nu, e_{i}\right\rangle\left\langle\nu, e_{j}\right\rangle\langle B \nu, \nu\rangle=\langle A \nu, \nu\rangle\left(\lambda_{j}\left\langle\nu, e_{j}\right\rangle\left\langle\nu, B e_{i}\right\rangle+\lambda_{i}\left\langle\nu, e_{i}\right\rangle\left\langle\nu, B e_{j}\right\rangle\right)
$$

for all $\nu \in \mathbb{R}^{d} \backslash\{0\}$ and all $i, j \in\{1, \ldots, d\}$. With $\nu=e_{i} \neq e_{j}$ we obtain from (A.2) the condition $0=\lambda_{i}^{2}\left\langle e_{i}, B e_{j}\right\rangle$. Since $\lambda_{i} \neq 0$, it follows that

$$
B:\left(e_{i} \otimes e_{j}\right)=B:\left(e_{j} \otimes e_{i}\right)=\left\langle e_{i}, B e_{j}\right\rangle=0=A:\left(e_{i} \otimes e_{j}\right)
$$

for all $i \neq j$. Assume again that $i \neq j$. With the choice $\nu=a_{i} e_{i}+a_{j} e_{j}$, where $a_{i}^{2}+a_{j}^{2}=1$ and $a_{i} a_{j} \neq 0$, it follows from (A.2) in combination with (A.3) that

$$
a_{i}^{2}\left(\left\langle B e_{i}, e_{i}\right\rangle-\lambda_{i} c_{i j}\right)+a_{j}^{2}\left(\left\langle B e_{j}, e_{j}\right\rangle-\lambda_{j} c_{i j}\right)=0
$$


for all these $a_{i}$ and $a_{j}$. Here, $c_{i j}=\left(2 \lambda_{i} \lambda_{j}\right)^{-1}\left(\lambda_{j}\left\langle B e_{i}, e_{i}\right\rangle+\lambda_{i}\left\langle B e_{j}, e_{j}\right\rangle\right)$. This implies that $\left\langle B e_{i}, e_{i}\right\rangle-\lambda_{i} c_{i j}=0$ for all $i \neq j$ from which we deduce (with $j=1$ ) that

$$
\left\langle B e_{i}, e_{i}\right\rangle=\frac{\left\langle B e_{1}, e_{1}\right\rangle}{\lambda_{1}}\left\langle A e_{i}, e_{i}\right\rangle
$$

for all $i \in\{1, \ldots, d\}$. Together with (A.3) it follows that $B=\frac{\left\langle B e_{1}, e_{1}\right\rangle}{\lambda_{1}} A$.

Lemma A.2. Let $A, B \in \operatorname{Lin}\left(\mathbb{R}^{d}, \mathbb{R}^{d}\right)$ be symmetric with $\operatorname{det} A>0$ and $\operatorname{det} B>0$. Assume that for all $\nu \in \mathbb{R}^{d} \backslash\{0\}$ we have $-R_{\nu} \mathcal{K}=\mathcal{K}$, where $\mathcal{K}=\left\{\eta \in \mathbb{R}^{d} ;\langle B \eta, \eta\rangle \leq 1\right\}$ and $R_{\nu}=\mathbb{I}-\frac{2}{\langle A \nu, \nu\rangle} A \nu \otimes \nu$. Then there exists $\beta>0$ such that $B=\beta A^{-1}$.

Proof. Short calculations show that

$$
\begin{aligned}
R_{\nu}^{\top} B R_{\nu} & =B+\frac{2}{\langle A \nu, \nu\rangle^{2}}(-\langle A \nu, \nu\rangle(B A \nu \otimes \nu+\nu \otimes B A \nu)+2\langle B A \nu, A \nu\rangle \nu \otimes \nu) \\
& =: B+\frac{2}{\langle A \nu, \nu\rangle^{2}} T_{\nu}
\end{aligned}
$$

The assumption $-R_{\nu} \mathcal{K}=\mathcal{K}$ implies that for all $\nu \in \mathbb{R}^{d} \backslash\{0\}$ and all $\eta \in \mathbb{R}^{d}$ we have

$$
\langle B \eta, \eta\rangle \leq 1 \Leftrightarrow\langle B \eta, \eta\rangle+\frac{2}{\langle A \nu, \nu\rangle^{2}}\left\langle T_{\nu} \eta, \eta\right\rangle \leq 1
$$

Thus, $\left\langle T_{\nu} \eta, \eta\right\rangle=0$ for all $\eta \in \mathbb{R}^{d}$. Note that

$$
\left\langle T_{\nu} \eta, \eta\right\rangle=2\langle\nu, \eta\rangle(\langle B A \nu, A \nu\rangle\langle\eta, \nu\rangle-\langle A \nu, \nu\rangle\langle B A \nu, \eta\rangle)
$$

Let $\left\{e_{1}, \ldots, e_{d}\right\}$ be an orthonormal basis of eigenvectors of $A$ with eigenvalues $\lambda_{i}>0$. Let furthermore $\nu=e_{i}+\alpha e_{j}$ and $\eta=e_{i}$ for $i \neq j$ and $\alpha \in \mathbb{R}$. From $\left\langle T_{\nu} \eta, \eta\right\rangle=0$ it follows that for all $\alpha \in \mathbb{R}$ we have

$$
0=\alpha \lambda_{i} \lambda_{j}\left\langle B e_{i}, e_{j}\right\rangle+\alpha^{2} \lambda_{j}\left(\lambda_{j}\left\langle B e_{j}, e_{j}\right\rangle-\lambda_{i}\left\langle B e_{i}, B e_{i}\right\rangle\right)-\alpha^{3} \lambda_{j}^{2}\left\langle B e_{j}, e_{i}\right\rangle .
$$

This implies that $\left\langle B e_{i}, e_{j}\right\rangle=0$ for $i \neq j$ and $\lambda_{j}\left\langle B e_{j}, e_{j}\right\rangle=\lambda_{i}\left\langle B e_{i}, B e_{i}\right\rangle$ for all $i, j$, from which we conclude that $\left\langle B e_{j}, e_{j}\right\rangle=\lambda_{1}\left\langle B e_{1}, e_{1}\right\rangle \lambda_{j}^{-1}=\lambda_{1}\left\langle B e_{1}, e_{1}\right\rangle\left\langle A^{-1} e_{j}, e_{j}\right\rangle$. In the same way as in the proof of the previous Lemma, it follows finally that $B=\lambda_{1}\left\langle B e_{1}, e_{1}\right\rangle A^{-1}$.

\section{References}

[AC00] Jochen Alberty and Carsten Carstensen. Numerical analysis of time-depending primal elastoplasticity with hardening. SIAM J. Numer. Anal., 37(4):1271-1294, 2000. 
[AC04] H.-D. Alber and K. Chełmiński. Quasistatic problems in viscoplasticity theory I: Models with linear hardening. In I. Gohberg et al., editor, Operator theoretical methods and applications to mathematical physics. The Erhard Meister memorial volume, volume 147 of Oper. Theory, Adv. Appl., pages 105-129. Birkhäuser, Basel, 2004.

[Alb98] H.-D. Alber. Materials with memory. Initial-boundary value problems for constitutive equations with internal variables, volume 1682 of Lecture Notes in Mathematics. Springer-Verlag, Berlin, 1998.

[AN09] H.-D. Alber and S. Nesenenko. Local $H^{1}$-regularity and $H^{1 / 3-\delta}$-regularity up to the boundary in time dependent viscoplasticity. Asymtotic Analysis, 63(3):151-187, 2009.

[BF96] A. Bensoussan and J. Frehse. Asymtotic behaviour of the time dependent NortonHoff law in plasticiy theory and $H_{l o c}^{1}$ regularity. Comment. Math. Univ. Carolinae, 37(2):285-304, 1996.

[Bré73] H. Brézis. Opérateurs maximaux monotones et semi-groupes de contractions dans les espaces de Hilbert. North-Holland Mathematics Studies, 1973.

[Dem08] A. Demyanov. Quasistatic evolution in the theory of elasto-plastic plates. Part II: Regularity of bending moments. Preprint no. 42/2008/M, SISSA Trieste, 2008.

[Dem09] A. Demyanov. Regularity of stresses in Prandtl-Reuss perfect plasticity. Calc. Var. Partial Differ. Equ., 34(1):23-72, 2009.

[DL72] G. Duvaut and J. L. Lions. Les inéquations en mécanique et en physique, volume 21 of Travaux et recherches mathematiques. Dunod, Paris, 1972.

[FL08a] J. Frehse and D. Löbach. Hölder continuity for the displacements in isotropic and kinematic hardening with von Mises yield criterion. Z. Angew. Math. Mech., 88(8):617$629,2008$.

[FL08b] J. Frehse and D. Löbach. Regularity results for three dimensional isotropic and kinematic hardening including boundary differentiability. Preprint SFB611 432, University of Bonn, 2008.

[GH96] M. Giaquinta and S. Hildebrandt. Calculus of Variations I. Springer-Verlag, Berlin, Heidelberg, 1996.

[Gri85] P. Grisvard. Elliptic Problems in Nonsmooth Domains. Pitman Publishing Inc, Boston, 1985. 
[HHLN88] J. Haslinger, I. Hlaváček, J. Lovíšek, and J. Nečas. Solution of variational inequalities in mechanics, volume 66 of Applied Mathematical Sciences. Springer-Verlag, New York, 1988.

[HR99] W. Han and B. D. Reddy. Plasticity, Mathematical Theorie and Numerical Analysis. Springer Verlag Inc., New York, 1999.

[IT79] A.D. Ioffe and V.M. Tihomirov. Theory of extremal problems. Translated from the Russian by K. Makowski. Studies in Mathematics and its Applications, Vol. 6. Amsterdam, New York, Oxford: North-Holland Publishing Company., 1979.

[Joh78] C. Johnson. On plasticity with hardening. J. Math. Anal. Appl., 62:325-336, 1978.

[KM08] D. Knees and A. Mielke. Energy release rate for cracks in finite-strain elasticity. Math. Methods Appl. Sci., 31(5):501-528, 2008.

[KN08] D. Knees and P. Neff. Regularity up to the boundary for nonlinear elliptic systems arising in time-incremental infinitesimal elasto-plasticity. SIAM J. Math. Anal., 40(1):21-43, 2008.

[Kne06] D. Knees. Global regularity of the elastic fields of a power-law model on Lipschitz domains. Math. Methods Appl. Sci., 29:1363-1391, 2006.

[Kne09] D. Knees. On global spatial regularity in elasto-plasticity with linear hardening. Calc. Var. Partial Differ. Equ., 36:611-625, 2009.

[Kre99] P. Krejčí. Evolution variational inequalities and multidimensional hysteresis operators. Drábek, Pavel (ed.) et al., Nonlinear differential equations. Proceedings of talks given at the seminar in differential equations, Chvalatice, Czech Republic, June 29-July 3, 1998. Boca Raton, FL: Chapman \&amp; Hall/CRC. Chapman Hall/CRC Res. Notes Math. 404, 47-110 (1999)., 1999.

[Mie05] A. Mielke. Evolution of rate-independent systems (ch. 6). In C.M. Dafermos and E. Feireisl, editors, Handbook of Differential Equations, Evolutionary Equations II, pages 461-559. Elsevier B.V., 2005.

[MPPS09] A. Mielke, L. Paoli, A. Petrov, and U. Stefanelli. Error estimates for space-time discretizations of a rate-independent variational inequality. WIAS-Preprint No. 1407, WIAS Berlin, 2009.

[MRS08] A. Mielke, T. Roubíček, and U. Stefanelli. T-limits and relaxations for rateindependent evolutionary problems. Calc. Var. Partial Differ. Equ., 31(3):387-416, 2008 . 
[NC05] P. Neff and K. Chełmiński. Infinitesimal elastic-plastic Cosserat micropolar theory. Modelling and global existence in the rate independent case. Proc. Roy. Soc. Edinburgh Sec. A, 135:1017-1039, 2005.

[NC08] P. Neff and K. Chełmiński. $H_{l o c}^{1}$-stress and strain regularity in cosserat plasticity. Technical report, Technical University of Darmstadt, 2008. (submitted to ZAMM).

[Rep96] S. I. Repin. Errors of finite element method for perfectly elasto-plastic problems. Math. Models Methods Appl. Sci., 6(5):587-604, 1996.

[Ser92] G.A. Seregin. Differential properties of solutions of evolutionary variational inequalities in plasticity theory. Probl. Mat. Anal., 12:153-173, 1992.

[Ser99] G. A. Seregin. Remarks on the regularity up to the boundary for solutions to variational problems in plasticity theory. J. Math. Sci., 93(5):779-783, 1999.

[Shi99] P. Shi. Interior regularity of solutions to a dynamic cyclic plasticity model in higher dimensions. Adv. Math. Sci. Appl., 9(2):817-837, 1999.

[Tri83] H. Triebel. Theory of function spaces, volume 78 of Monographs in Mathematics. Birkhäuser, Basel, 1983. 